\title{
The Generalized Additive Weibull-G Family of Distributions
}

\author{
Amal S. Hassan ${ }^{1}$, Saeed E. Hemeda ${ }^{1}$, Sudhansu S. Maiti ${ }^{2} \&$ Sukanta Pramanik $^{3}$ \\ ${ }^{1}$ Institute of Statistical Studies \& Research, Cairo University, Egypt \\ 2 Department of Statistics, Visva-Bharati University, Santiniketan-731 235, India \\ ${ }^{3}$ Department of Statistics, Siliguri College, North Bengal University, Siliguri-734 001, India \\ Correspondence: Saeed E. Hemeda, Institute of Statistical Studies \& Research, Cairo University, Egypt. \\ E-mail: saidhemeda25jan@gmail.com
}

Received: April 20, 2017 Accepted: July 1, 2017 Online Published: August 3, 2017

doi:10.5539/ijsp.v6n5p65

URL: https://doi.org/10.5539/ijsp.v6n5p65

\begin{abstract}
In this paper, we present a new family, depending on additive Weibull random variable as a generator, called the generalized additive Weibull generated-family (GAW-G) of distributions with two extra parameters. The proposed family involves several of the most famous classical distributions as well as the new generalized Weibull-G family which already accomplished by Cordeiro et al. (2015). Four special models are displayed. The expressions for the incomplete and ordinary moments, quantile, order statistics, mean deviations, Lorenz and Benferroni curves are derived. Maximum likelihood method of estimation is employed to obtain the parameter estimates of the family. The simulation study of the new models is conducted. The efficiency and importance of the new generated family is examined through real data sets.
\end{abstract} Keywords: Additive Weibull, Mean deviation, Moments, Estimation

\section{Introduction}

In recent years, the generated families of probability distributions have been broadly utilized for modeling real-data in many applied areas. These generated families are defined by adding one or more parameters to the baseline model. The generated families generalized and extended most of the formal distributions. Some of the generators are the beta-G by Eugene et al. (2002), gamma-G by Zografos and Balakrishanan (2009), Kumaraswamy-G by Cordeiro and de Castro (2011), generalized beta-G by Alexander et al. (2012), transformed-transformer (T-X) by Alzaatreh et al. (2013), WeibullG by Bourguignon (2014), type I half-logistic-G by Cordeiro et al. (2016), additive Weibull-G by Hassan and Hemeda (2016) among others.

A more general family called the generalized Weibull-G family (GW-G) of distributions was introduced by Cordeiro et al. (2015). They considered a baseline cumulative distribution function (cdf) $\mathrm{G}(\mathrm{x} ; \xi)$, the probability density function (pdf) $\mathrm{g}(\mathrm{x} ; \xi)$ with parameter vector $\xi$ and the Weibull distribution as a generator. They defined the cdf and pdf of the GW-G family as follows

$$
\begin{gathered}
F(x ; a, b, \xi)=\int_{0}^{-\ln [\bar{G}(x ; \xi)]} a b t^{b-1} e^{-a t^{b}} d t=1-e^{-a\{-\ln [\bar{G}(x ; \xi)]\}^{b}} ; x \geq 0, a, b>0 . \\
f(x ; a, b, \xi)=\frac{a b g(x ; \xi)}{\bar{G}(x ; \xi)}\{-\ln [\bar{G}(x ; \xi)]\}^{b-1} e^{-a\{-\ln [\bar{G}(x ; \xi)]\}^{b}} ; x \geq 0, a, b>0 .
\end{gathered}
$$

where, $\bar{G}(x ; \xi)=1-G(x ; \xi)$

This article aims to introduce a new family of distribution called the GAW-G, which includes GW-G family as a special case, besides it contains several of the existing probability distributions. The current article can be arranged as follows. The GAW generated family of distributions is formulated in Section 2. Four special models of GAW-G family are displayed in Section 3. Some structural properties of the GAW-G family are provided in Section 4. In Section 5, maximum likelihood estimators of the model parameters are derived. In Section 6, Simulation study results of four special models have been reported. In Section 7, an illustrative application based on a real data is investigated. A conclusion is provided in Section 8.

\section{The Generalized Additive Weibull-G Family}

In this section, we define a generalized additive Weibull generated family of continuous distributions by using the additive Weibull random variable as a generator. The reliability and hazard rate functions are defined and discussed analytically. Furthermore, the asymptotic of pdf, cdf and hazard function is explained. According to Lemonte et al. (2014), the cdf and 
pdf of AW distribution with shape parameters b, $d$ and scale parameters a, c are given, respectively, by

$$
\begin{gathered}
F(x ; a, b, c, d)=1-e^{-c x^{d}-a x^{b}} ; x \geq 0, a, b, c, d>0 . \\
f(x ; a, b, c, d)=\left(c d x^{d-1}+a b x^{b-1}\right) e^{-c x^{d}-a x^{b}} ; x \geq 0, a, b, c, d>0 .
\end{gathered}
$$

To obtain the cdf of GAWCG, replacing the Weibull generator defined in (1) by the additive Weibull generator defined in (4) as the following

$$
\begin{aligned}
F(x ; \Phi) & =\int_{0}^{-\ln [\bar{G}(x ; \xi)]}\left(c d t^{d-1}+a b t^{b-1}\right) e^{-c t^{d}-a t^{b}} d t \\
& =1-\exp \left\{-c[-\ln [\bar{G}(x ; \xi)]]^{d}-a[-\ln [\bar{G}(x ; \xi)]]^{b}\right\} ; x \geq 0, a, b, c, d>0 .
\end{aligned}
$$

where $\Phi=(a, b, c, d, \xi)$ and $\bar{G}(x ; \xi)=1-G(x ; \xi)$. The corresponding GAW-G pdf takes the following form

$$
\begin{aligned}
f(x ; \Phi)= & \frac{g(x ; \xi)}{\bar{G}(x ; \xi)}\left\{c d[-\ln [\bar{G}(x ; \xi)]]^{d-1}+a b[-\ln [\bar{G}(x ; \xi)]]^{b-1}\right\} \\
& . \exp \left\{-c[-\ln [\bar{G}(x ; \xi)]]^{d}-a[-\ln [\bar{G}(x ; \xi)]]^{b}\right\} ; x \geq 0, a, b, c, d>0 .
\end{aligned}
$$

A random variable having GAW-G density function (6) will be denoted by $X \sim G A W-G(x ; \Phi)$. Note that, for a $=0$ or $\mathrm{c}=0$ the pdf (6) reduces to the GAW-G family defined by Cordeiro et al.(2015). Also, we obtain the same result for $b=d$, with scale parameter $a+c$.

Furthermore, the reliability and the hazard rate functions of GAW-G family are given, respectively, by

$$
R(x ; \Phi)=e^{-c[-\ln [\bar{G}(x ; \xi)]]^{d}-a[-\ln [\bar{G}(x ; \xi)]]^{b}}
$$

The hazard rate function of GAW-G family is

$$
h(x ; \Phi)=\frac{g(x ; \xi)}{\bar{G}(x ; \xi)}\left\{c d[-\ln [\bar{G}(x ; \xi)]]^{d-1}+a b[-\ln [\bar{G}(x ; \xi)]]^{b-1}\right\}
$$

Here is a description regarding analytical behaviour of GAW-G family. The critical points of this family are the roots of the equation

$$
\frac{d}{d x} h(x ; \Phi)=h^{\prime}(x ; \Phi)=0
$$

where

$$
\begin{gathered}
h^{\prime}(x ; \Phi)=\frac{g(x ; \xi)}{\bar{G}(x ; \xi)}\left[\frac{c d(d-1) g(x ; \xi)}{\bar{G}(x ; \xi)}\{-\ln \bar{G}(x ; \xi)\}^{d-2}+\frac{a b(b-1) g(x ; \xi)}{\bar{G}(x ; \xi)}\{-\ln \bar{G}(x ; \xi)\}^{b-2}\right] \\
+\left[\left\{\frac{g(x ; \xi)}{\bar{G}(x ; \xi)}\right\}^{2}+\frac{g^{\prime}(x ; \xi)}{\bar{G}(x ; \xi)}\right]\left[c d\{-\ln \bar{G}(x ; \xi)\}^{d-1}+a b\{-\ln \bar{G}(x ; \xi)\}^{b-1}\right] \\
\text { i.e.h }(x ; \Phi)=\left[\frac{g(x ; \xi)}{\bar{G}(x ; \xi)}\right]^{2}\left[c d(d-1)\{-\ln \bar{G}(x ; \xi)\}^{d-2}+a b(b-1)\{-\ln \bar{G}(x ; \xi)\}^{b-2}\right] \\
+\left[\frac{g^{\prime}(x ; \xi)}{\bar{G}(x ; \xi)}\right]\left[c d\{-\ln \bar{G}(x ; \xi)\}^{d-1}+a b\{-\ln \bar{G}(x ; \xi)\}^{b-1}\right]
\end{gathered}
$$

There is more than one root to this equation. When $b=1$, it becomes to some extent a simpler model and then $x=x_{0}$ is a root of the equation

$c d(d-1)\left[\frac{g(x ; \xi)}{\bar{G}(x ; \xi)}\right]^{2}\{-\ln \bar{G}(x ; \xi)\}^{d-2}+\frac{g^{\prime}(x ; \xi)}{\bar{G}(x ; \xi)}\left[c d\{-\ln \bar{G}(x ; \xi)\}^{d-1}+a\right]=0$.

When $b=d=1$,

$h^{\prime}(x ; \Phi)=-(c+a) \frac{g^{\prime}(x ; \xi)}{\bar{G}(x ; \xi)}=0$. 
Therefore, $x=x_{0}$ is a root of the equation

$g^{\prime}(x ; \xi)=0$,

and

$h^{\prime \prime}(x ; \Phi)=(c+a)\left\{\frac{g^{\prime \prime}(x ; \xi)}{\bar{G}(x ; \xi)}+\frac{g^{\prime}(x ; \xi) g(x ; \xi)}{\bar{G}^{2}(x ; \xi)}\right\}$.

The critical point $x_{0}$ which refers to a local maximum if $h^{\prime \prime}(x ; \Phi)>0(<0), \forall x<x_{0}$ and a local minimum if $h^{\prime \prime}(x ; \Phi)>0(<$ $0), \forall x>x_{0}$. It gives an inflexion point if either $h^{\prime \prime}(x ; \Phi)>0, \forall x \neq x_{0}$ or $h^{\prime \prime}(x ; \Phi)<0, \forall x \neq x_{0}$, where $h^{\prime \prime}(x ; \Phi)=\frac{d^{2} h(x ; \Phi)}{d x^{2}}$.

\section{Some Special Models for GAW-G Family}

In this section, some new special distributions, namely, GAW-uniform, GAW-Burr XII, and GAW-log logistic are introduced.

\subsection{GAW-uniform Distribution}

Consider the baseline distribution is uniform on the interval $(0, \theta), \theta>0$ with the pdf and cdf, respectively

$$
g(x ; \theta)=\frac{1}{\theta} ; 0<x<\theta<\infty, G(x, \theta)=\frac{x}{\theta} .
$$

The cdf of GAW-uniform (GAWU) distribution is obtained by substituting the pdf and cdf of uniform in (5) as follows

$$
F(x ; a, b, c, d, \theta)=1-\exp \left\{-c\left[-\ln \left(\frac{\theta-x}{\theta}\right)\right]^{d}-a\left[-\ln \left(\frac{\theta-x}{\theta}\right)\right]^{b}\right\} ; 0<x<\theta<\infty .
$$

The corresponding pdf is given by

$$
\begin{aligned}
f(x ; a, b, c, d, \theta)= & \frac{1}{(\theta-x)}\left\{c d\left[-\ln \left(\frac{\theta-x}{\theta}\right)\right]^{d-1}+a b\left[-\ln \left(\frac{\theta-x}{\theta}\right)\right]^{b-1}\right\} \\
& . \exp \left\{-c\left[-\ln \left(\frac{\theta-x}{\theta}\right)\right]^{d}-a\left[-\ln \left(\frac{\theta-x}{\theta}\right)\right]^{b}\right\} ; 0<x<\theta<\infty, a, b, c, d>0 .
\end{aligned}
$$

The survival and hazard rate functions are given respectively as follows

$$
\begin{gathered}
R(x ; a, b, c, d, \theta)=\exp \left\{-c\left[-\ln \left(\frac{\theta-x}{\theta}\right)\right]^{d}-a\left[-\ln \left(\frac{\theta-x}{\theta}\right)\right]^{b}\right\} \\
h(x ; a, b, c, d, \theta)=\frac{1}{(\theta-x)}\left\{c d\left[-\ln \left(\frac{\theta-x}{\theta}\right)\right]^{d-1}+a b\left[-\ln \left(\frac{\theta-x}{\theta}\right)\right]^{b-1}\right\}
\end{gathered}
$$

\subsection{GAW-Gumbel Distribution}

Consider the Gumbel distribution with location parameter $\lambda \in R$ and scale parameter $v>0$ where the pdf and cdf for $(\lambda \in R)$ are

$$
g(x ; \lambda, v)=\frac{1}{v} \exp \left\{\left(\frac{x-\lambda}{v}\right)-\exp \left(\frac{x-\lambda}{v}\right)\right\}
$$

and

$$
G(x ; \lambda, v)=1-\exp \left\{-\exp \left(\frac{x-\lambda}{v}\right)\right\}
$$

Inserting these equations into (5) and (6), the pdf and cdf of the GAW-Gumbel distribution will be obtained as follows

$$
\begin{aligned}
F(x ; a, b, c, d, \lambda, v)= & 1-\exp \left\{-c\left[-\ln \left(\exp \left(-\exp \left(\frac{x-\lambda}{v}\right)\right)\right)\right]^{d}-a\left[-\ln \left(\exp \left(-\exp \left(\frac{x-\lambda}{v}\right)\right)\right)\right]^{b}\right\} \\
f(x ; a, b, c, d, \lambda, v)= & \frac{1}{v} \exp \left(\frac{x-\lambda}{v}\right)\left\{c d\left[-\ln \left(\exp \left(-\exp \left(\frac{x-\lambda}{v}\right)\right)\right)\right]^{d-1}\right. \\
& \left.+a b\left[-\ln \left(\exp \left(-\exp \left(\frac{x-\lambda}{v}\right)\right)\right)\right]^{b-1}\right\} \exp \left\{-c\left[-\ln \left(\exp \left(-\exp \left(\frac{x-\lambda}{v}\right)\right)\right)\right]^{d}\right. \\
& \left.-a\left[-\ln \left(\exp \left(-\exp \left(\frac{x-\lambda}{v}\right)\right)\right)\right]^{b}\right\} ; x, a, b, c, d, \lambda, v>0 .
\end{aligned}
$$


The survival and hazard rate functions are given respectively as follows

$$
\begin{gathered}
R(x ; a, b, c, d, \lambda, v)=\exp \left\{-c\left[-\ln \left(\exp \left(-\exp \left(\frac{x-\lambda}{v}\right)\right)\right)\right]^{d}-a\left[-\ln \left(\exp \left(-\exp \left(\frac{x-\lambda}{v}\right)\right)\right)\right]^{b}\right\} \\
h(x ; a, b, c, d, \lambda, v)=\frac{1}{v} \exp \left(\frac{x-\lambda}{v}\right)\left\{c d\left[-\ln \left(\exp \left(-\exp \left(\frac{x-\lambda}{v}\right)\right)\right)\right]^{d-1}\right. \\
\left.+a b\left[-\ln \left(\exp \left(-\exp \left(\frac{x-\lambda}{v}\right)\right)\right)\right]^{b-1}\right\}
\end{gathered}
$$

\subsection{GAW log-logistic Distribution}

Assuming that the baseline distribution is log-logistic (see (Bennett (1983)) with the following pdf and cdf,

$$
g(x ; \lambda, \alpha)=\alpha \lambda^{-\alpha} x^{\alpha-1}\left[1+\left(\frac{x}{\lambda}\right)^{\alpha}\right]^{-2}
$$

and

$$
G(x ; \lambda, \alpha)=1-\left[1+\left(\frac{x}{\lambda}\right)^{\alpha}\right]^{-1}
$$

As previously mentioned, the cdf and pdf of the generalized additive Weibull log -logistic(GAWLL) distribution are obtained by substituting the previous pdf and cdf in (5) and (6) as follows

$$
\begin{aligned}
F(x ; a, b, c, d, \lambda, \alpha)=1- & \exp \left\{-c\left[\ln \left(1+\left(\frac{x}{\lambda}\right)^{\alpha}\right)\right]^{d}-a\left[\ln \left(1+\left(\frac{x}{\lambda}\right)^{\alpha}\right)\right]^{b}\right\} ; x \geq 0, \lambda, \alpha>0, \\
f(x ; a, b, c, d, \lambda, \alpha)= & \frac{\alpha \lambda^{-\alpha} x^{\alpha-1}}{\left(1+\left(\frac{x}{\lambda}\right)^{\alpha}\right)}\left\{c d\left[\ln \left(1+\left(\frac{x}{\lambda}\right)^{\alpha}\right)\right]^{d-1}+a b\left[\ln \left(1+\left(\frac{x}{\lambda}\right)^{\alpha}\right)\right]^{b-1}\right\} \\
& \quad \cdot \exp \left\{-c\left[\ln \left(1+\left(\frac{x}{\lambda}\right)^{\alpha}\right)\right]^{d}-a\left[\ln \left(1+\left(\frac{x}{\lambda}\right)^{\alpha}\right)\right]^{b}\right\} ; x>0 .
\end{aligned}
$$

The survival and hazard rate functions take, respectively, the following forms

$$
\begin{gathered}
R(x ; a, b, c, d, \lambda, \alpha)=\exp \left\{-c\left[\ln \left(1+\left(\frac{x}{\lambda}\right)^{\alpha}\right)\right]^{d}-a\left[\ln \left(1+\left(\frac{x}{\lambda}\right)^{\alpha}\right)\right]^{b}\right\} \\
h(x ; a, b, c, d, \lambda, \alpha)=\frac{\alpha \lambda^{-\alpha} x^{\alpha-1}}{\left(1+\left(\frac{x}{\lambda}\right)^{\alpha}\right)}\left\{c d\left[\ln \left(1+\left(\frac{x}{\lambda}\right)^{\alpha}\right)\right]^{d-1}+a b\left[\ln \left(1+\left(\frac{x}{\lambda}\right)^{\alpha}\right)\right]^{b-1}\right\}
\end{gathered}
$$

\subsection{GAW-Burr XII Distribution}

Considering the baseline distribution is Burr XII (see Burr (1942)) with the following pdf and cdf

$$
\begin{gathered}
g(x ; \alpha, \theta)=\alpha \theta x^{\alpha-1}\left(1+x^{\alpha}\right)^{-(\theta+1)} ; x \geq 0, \alpha, \theta>0, \\
G(x ; \alpha, \theta)=1-\left(1+x^{\alpha}\right)^{-\theta} ; x \geq 0, \alpha, \theta>0 .
\end{gathered}
$$

The cdf of GAW-Burr XII (GAWBXII) distribution is obtained by substituting the pdf and cdf of Burr-XII in (5) and (6) as follows

$$
F(x ; a, b, c, d, \alpha, \theta)=1-\exp \left\{-c \theta^{d}\left[\ln \left(1+x^{\alpha}\right)\right]^{d}-a \theta^{b}\left[\ln \left(1+x^{\alpha}\right)\right]^{b}\right\} ; x>0 .
$$

The corresponding pdf is

$$
\begin{aligned}
f(x ; a, b, c, d, \alpha, \theta)= & \frac{\alpha \theta x^{\alpha-1}}{\left(1+x^{\alpha}\right)}\left\{c d \theta^{d-1}\left[\ln \left(1+x^{\alpha}\right)\right]^{d-1}+a b \theta^{b-1}\left[\ln \left(1+x^{\alpha}\right)\right]^{b-1}\right\} \\
& . \exp \left\{-c \theta^{d}\left[\ln \left(1+x^{\alpha}\right)\right]^{d}-a \theta^{b}\left[\ln \left(1+x^{\alpha}\right)\right]^{b}\right\} ; x>0, a, b, c, d, \alpha, \theta>0
\end{aligned}
$$


The survival and hazard rate functions are obtained, respectively, as follows

$$
\begin{gathered}
R(x ; a, b, c, d, \alpha, \theta)=\exp \left\{-c \theta^{d}\left[\ln \left(1+x^{\alpha}\right)\right]^{d}-a \theta^{b}\left[\ln \left(1+x^{\alpha}\right)\right]^{b}\right\}, \\
h(x ; a, b, c, d, \alpha, \theta)=\frac{\alpha \theta x^{\alpha-1}}{\left(1+x^{\alpha}\right)}\left\{c d \theta^{d-1}\left[\ln \left(1+x^{\alpha}\right)\right]^{d-1}+a b \theta^{b-1}\left[\ln \left(1+x^{\alpha}\right)\right]^{b-1}\right\} .
\end{gathered}
$$

Plots of pdf and hazard rate function for some parameter values for the selected distributions are represented through Figure 1.

From Figure 1, it appears that the shape of the distribution depend heavily on parameter values. In fact, the shape could be left skewed, symmetric and right skewed, which will depend on the values of the parameter. Thus this distribution could be suitable to model many kind of data.

\section{Some Mathematical Properties}

In this section, some general results of the GAW-G family are derived.

\subsection{Mixture Presentation}

Expansion formulas for the cdf and pdf of the GAW-G family are derived. The power series for the following exponential function can be written as

$$
\begin{aligned}
\exp \left\{-c[-\ln [\bar{G}(x ; \xi)]]^{d}-a[-\ln [\bar{G}(x ; \xi)]]^{b}\right\} & =\sum_{i=0}^{\infty} \frac{(-1)^{i}}{i !}\left[c[-\ln [\bar{G}(x ; \xi)]]^{d}+a[-\ln [\bar{G}(x ; \xi)]]^{b}\right]^{i} \\
& =\sum_{i=0}^{\infty} \frac{(-1)^{i} c^{i}}{i !}\left[[-\ln [\bar{G}(x ; \xi)]]^{i d}\left[1+\frac{a}{c}[-\ln [\bar{G}(x ; \xi)]]^{b-d}\right]\right]^{i}
\end{aligned}
$$

Based on (9), the cdf in (5) will be

$$
\begin{gathered}
F(x ; \Phi)=1-\left\{1-\sum_{i=0}^{\infty} \frac{(-1)^{i} c^{i}}{i !}\left[[-\ln [\bar{G}(x ; \xi)]]^{i d}\left[1+\frac{a}{c}[-\ln [\bar{G}(x ; \xi)]]^{b-d}\right]\right]^{i}\right\} \\
F(x ; \Phi)=\sum_{i=0}^{\infty} \frac{(-1)^{i} c^{i}}{i !}\left[[-\ln [\bar{G}(x ; \xi)]]^{i d}\left[1+\frac{a}{c}[-\ln [\bar{G}(x ; \xi)]]^{b-d}\right]\right]^{i}
\end{gathered}
$$

Since

$$
\left[\left[1+\frac{a}{c}[-\ln [\bar{G}(x ; \xi)]]^{b-d}\right]\right]^{i}=\sum_{j=0}^{\infty} \frac{i !}{j !(i-j) !}\left[\frac{a}{c}\right]^{j}[-\ln [\bar{G}(x ; \xi)]]^{j(b-d)}
$$

Substituting (11) into (10) then, the expansion for the cdf of GAW-G family can be written as

$$
F(x ; \Phi)=\sum_{i, j=0}^{\infty} \frac{(-1)^{i} a^{j} c^{i-j}}{j !(i-j) !}[-\ln [\bar{G}(x ; \xi)]]^{d(i-j)+b j}
$$

Based on the following expansion

$$
[-\ln (1-x)]^{c}=x^{c}+c \sum_{m=0}^{\infty} p_{m}(c+m) x^{m+c+1} ; c \in R \text { and } x \in(0,1),
$$

where $p_{m}(w)$ are Stirling coefficients and $\mathrm{w}=(\mathrm{c}+\mathrm{m})$. The first three coefficients are $p_{0}(w)=1, p_{1}(w)=\left(w+w^{2}\right) / 48, p_{2}(w)=$ $\left(-8-10 w+15 w^{2}+15 w^{3}\right) / 5760$. These coefficients are related to the Stirling polynomials by

$p_{n-1}(w)=S_{n}(w) /[n !(w+1)]$ for $n \geq 1$, where $S_{0}(w)=1, S_{1}(w)=(w+1) / 2, S_{3}(w)=w(w+1)^{2} / 24$.

See in details, Ward (1934), Flajonet and Odlyzko(1990), 


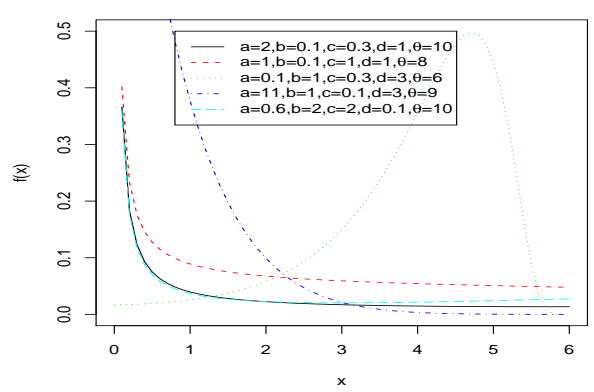

(a) GAW-Uniform distributions for different parameters

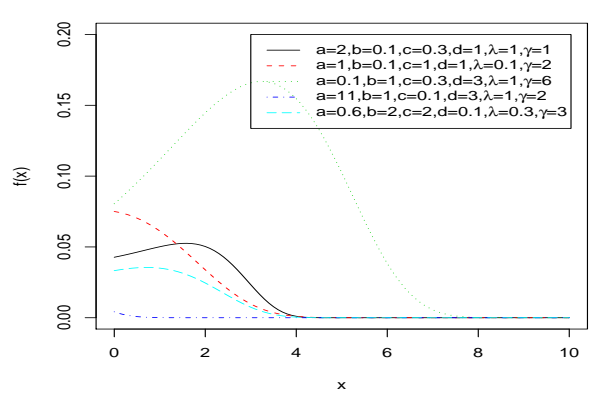

(c) GAW-Gumble distributions for parameters

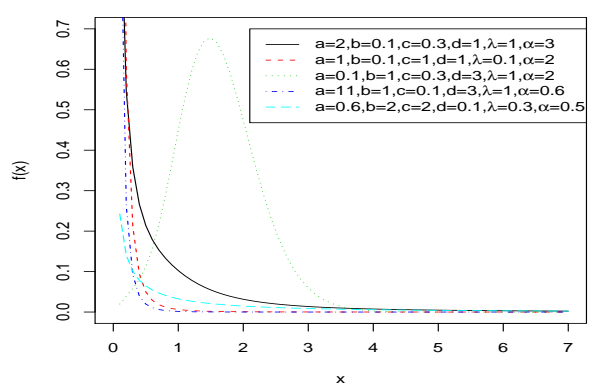

(e) GAW-Log-logistic distributions for parameters

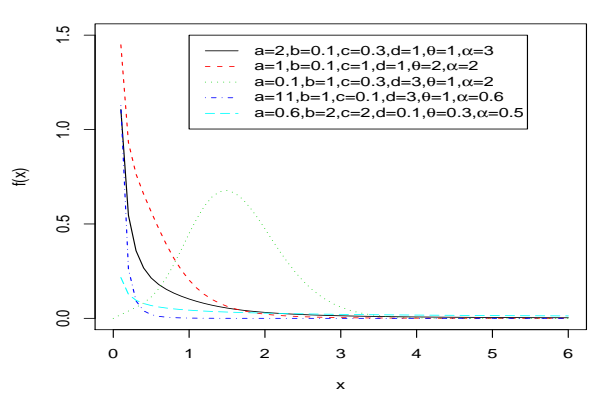

(g) GAW-Burr XII distributions for parameters



(b) Hazard rate of corresponding distributions



(d) Hazard rate of corresponding distributions



(f) Hazard rate of corresponding distributions



(h) Hazard rate of corresponding distributions

Figure 1. Graph of Generalized Additive Weibull distributions and their corresponding hazard rates. 
The formula in (13) holds for $m \geq 1$. Therefore, we can write

$$
\begin{aligned}
{[-\ln (1-G(x ; \xi))]^{d(i-j)+b j}=} & {[G(x ; \xi)]^{d(i-j)+b j}+[d(i-j)+b j] } \\
& \cdot \sum_{m=0}^{\infty} p_{m}[d(i-j)+b j+m][G(x ; \xi)]^{d(i-j)+b j+m+1}
\end{aligned}
$$

Substituting (14) into (12) the distribution function of GAW-G family will be

$$
\begin{aligned}
F(x ; \Phi)= & \sum_{i, j=0}^{\infty} \frac{(-1)^{i} a^{j} c^{i-j}}{j !(i-j) !}\left\{[G(x ; \xi)]^{d(i-j)+b j}+[d(i-j)+b j]\right. \\
& \left.\cdot \sum_{m=0}^{\infty} p_{m}[d(i-j)+b j+m][G(x ; \xi)]^{d(i-j)+b j+m}\right\}
\end{aligned}
$$

where $p_{m}(d(i-j)+b j+m)$ are the coefficients of the Stirling polynomials.

Expanding $[G(x ; \xi)]^{d(i-j)+b j}$ and $[G(x ; \xi)]^{d(i-j)+b j+m}$ in power series, $F(x ; \Phi)$ can be expressed as

$$
F(x ; \Phi)=\sum_{i, j=0}^{\infty} w_{i j}[G(x ; \xi)]^{k}+\sum_{i, j, m=0}^{\infty} w_{i j m}[G(x ; \xi)]^{k+m+1},
$$

where

$w_{i j}=\frac{(-1)^{i} a^{j} c^{i-j}}{j !(i-j) !}, w_{i j m}=\frac{(-1)^{i} a^{j} c^{i-j}[d(i-j)+b j] p_{m}[d(i-j)+b j+m]}{j !(i-j) !}, k=d(i-j)+b j$.

The corresponding pdf can be expressed as

$$
f(x ; \Phi)=\sum_{i, j=0}^{\infty} k w_{i, j}[G(x ; \xi)]^{k-1} g(x ; \xi)+\sum_{i, j, m=0}^{\infty}(k+m+1) w_{i, j, m}[G(x ; \xi)]^{k+m} g(x ; \xi)
$$

Another expression of the cdf of GAW-G family can be written as

$$
F(x ; \Phi)=\sum_{\epsilon}^{\infty} \eta_{\epsilon} H(x ; \xi),
$$

where $H(x ; \xi)$ denotes the cdf of the mixture exponential $[G(x ; \xi)]^{k}$ and $[G(x ; \xi)]^{k+m+1}$ distributions.

The corresponding pdf can be expressed as

$$
f(x ; \Phi)=\sum_{\epsilon}^{\infty} \eta_{\epsilon} h(x ; \xi)
$$

where $h(x ; \xi)$ is the pdf of the exp- $H(x ; \xi)$ distribution

\subsection{Quantile Function}

The quantile function, say $Q(u)=F^{-1}(u)$, of the GAW-G family is derived by inverting (5) as follows

$$
u=1-e^{-c\left[-\ln \left[1-x_{G}\right]\right]^{d}-a\left[-\ln \left[1-x_{G}\right]\right]^{b}} .
$$

After some simplifications, the previous equation is reduced to

$$
\ln (1-u)+c\left[-\ln \left[1-x_{G}\right]\right]^{d}+a\left[-\ln \left[1-x_{G}\right]\right]^{b}=0,
$$

where, $x_{G}=Q(u)$, and $u$ has the uniform distribution on interval $(0,1)$. Hence the nonlinear equation (20) is solved numerically to obtain the generated number of the random variable $X$.

\subsection{Moments}

The rth moment of random variable $\mathrm{X}$ can be obtained from pdf (17) as follows

$$
\begin{aligned}
\mu_{r}^{\prime} & =\int_{0}^{\infty} x^{r} f(x, \Phi) d x \\
& =\sum_{i, j=0}^{\infty} k w_{i, j} \int_{0}^{\infty} x^{r}[G(x ; \xi)]^{k-1} g(x ; \xi) d x+\sum_{i, j, m=0}^{\infty}(k+m+1) w_{i, j, m} \int_{0}^{\infty} x^{r}[G(x ; \xi)]^{k+m} g(x ; \xi) d x
\end{aligned}
$$


Therefore

$$
\mu_{r}^{\prime}=\sum_{i, j=0}^{\infty} w_{i j} I_{i, j, r}+\sum_{i, j, m=0}^{\infty} w_{i j m} I_{i, j, m, r} ; r=1,2, \ldots
$$

where, $I_{i, j, r}=\int_{0}^{\infty} k x^{r} h_{i, j}(x ; \xi) d x$ and $I_{i, j, m, r}=\int_{0}^{\infty}(k+m+1) x^{r} h_{i, j, m}(x ; \xi) d x$.

In particular, the mean and variance of GAW-G family are obtained as follows:

$$
E(X)=\sum_{i, j=0}^{\infty} w_{i j} I_{i, j, 1}+\sum_{i, j, m=0}^{\infty} w_{i j m} I_{i, j, m, 1}
$$

The variance is

$$
\operatorname{Var}(X)=\sum_{i, j=0}^{\infty} w_{i j} I_{i, j, 2}+\sum_{i, j, m=0}^{\infty} w_{i j m} I_{i, j, m, 2}-\left[\sum_{i, j=0}^{\infty} w_{i j} I_{i, j, 1}+\sum_{i, j, m=0}^{\infty} w_{i j m} I_{i, j, m, 1}\right]^{2}
$$

Additionally, measures of skewness and kurtosis of family can be obtained, based on (21), according to the following relations

$$
\begin{gathered}
\gamma_{1}=\frac{\mu_{3}^{\prime}-3 \mu_{2}^{\prime} \mu_{1}^{\prime}+2 \mu_{1}^{\prime 3}}{\left(\mu_{2}^{\prime}-\mu_{1}^{\prime 2}\right)^{3 / 2}} \\
\gamma_{2}=\frac{\mu_{4}^{\prime}-4 \mu_{3}^{\prime} \mu_{1}^{\prime}+6 \mu_{2}^{\prime} \mu_{1}^{\prime 2}-3 \mu_{1}^{\prime 4}}{\left(\mu_{2}^{\prime}-\mu_{1}^{\prime 2}\right)^{2}}
\end{gathered}
$$

Furthermore, the moment generating function of GAW-G family is as follows

$$
M_{X}(t)=\sum_{r=0}^{\infty} \frac{t^{r}}{r !} \mu_{r}^{\prime}
$$

where, $\mu_{r}^{\prime}$ is the $r^{\text {th }}$ moment about origin, then the moment generating function of GAW-G family is obtained by using (21) as follows

$$
M_{X}(t)=\sum_{r=0}^{\infty} \frac{t^{r}}{r !}\left[\sum_{i, j=0}^{\infty} w_{i j} I_{i, j, r}+\sum_{i, j, m=0}^{\infty} w_{i j m} I_{i, j, m, r}\right]
$$

\subsection{Distribution of Order Statistics}

Let $X_{1}, X_{2}, X_{3}, \ldots \ldots, X_{n}$ be a simple random sample from GAW-G family with cdf (5) and pdf (6) and $X_{1: n}, X_{2: n}, X_{3: n}, \ldots \ldots, X_{n: n}$ denote the corresponding order statistics. The pdf of $X_{r: n}$ is obtained through the following

$$
\begin{aligned}
& f_{r: n}(x ; \Phi)=\frac{1}{B(r, n-r+1)}[F(x ; \Phi)]^{r-1}[1-F(x ; \Phi)]^{n-r} f(x ; \Phi) . \\
& f_{r: n}(x ; \Phi)=\frac{1}{B(r, n-r+1)} \sum_{s=0}^{n-r}(-1)^{s}\left(\begin{array}{c}
n-r \\
s
\end{array}\right)[F(x ; \Phi)]^{r+s-1} f(x ; \Phi) .
\end{aligned}
$$

Using the cdf (5) and pdf (6), the pdf of rth order statistic from GAW-G family takes the following form

$$
\begin{aligned}
f_{r: n}(x ; \Phi)= & \frac{1}{B(r, n-r+1)} \sum_{s=0}^{n-r}(-1)^{s}\left(\begin{array}{c}
n-r \\
s
\end{array}\right)\left[1-\exp \left\{-c[-\ln [\bar{G}(x ; \xi)]]^{d}-a[-\ln [\bar{G}(x ; \xi)]]^{b}\right\}\right]^{r+s-1} \\
& \cdot \frac{g(x ; \xi)}{\bar{G}(x ; \xi)}\left\{c d[-\ln [\bar{G}(x ; \xi)]]^{d-1}+a b[-\ln [\bar{G}(x ; \xi)]]^{b-1}\right\} \\
& \cdot \exp \left\{-c[-\ln [\bar{G}(x ; \xi)]]^{d}-a[-\ln [\bar{G}(x ; \xi)]]^{b}\right\} .
\end{aligned}
$$


Since

$$
\begin{aligned}
{\left[1-\exp \left\{-c[-\ln [\bar{G}(x ; \xi)]]^{d}-a[-\ln [\bar{G}(x ; \xi)]]^{b}\right\}\right]^{r+s-1}=} & \sum_{w=0}^{\infty}(-1)^{w}\left(\begin{array}{c}
r+s-1 \\
w
\end{array}\right) \\
& \quad \exp \left\{-w c[-\ln [\bar{G}(x ; \xi)]]^{d}-w a[-\ln [\bar{G}(x ; \xi)]]^{b}\right\} .
\end{aligned}
$$

Substituting in (22), therefore

$$
\begin{aligned}
f_{r: n}(x ; \Phi)= & \frac{1}{B(r, n-r+1)} \sum_{w=0}^{\infty} \sum_{s=0}^{n-r}(-1)^{s+w}\left(\begin{array}{c}
n-r \\
s
\end{array}\right)\left(\begin{array}{c}
r+s-1 \\
w
\end{array}\right) \\
& \cdot \frac{g(x ; \xi)}{\bar{G}(x ; \xi)}\left\{c d[-\ln [\bar{G}(x ; \xi)]]^{d-1}+a b[-\ln [\bar{G}(x ; \xi)]]^{b-1}\right\} \\
& . \exp \left\{-c(w+1)[-\ln [\bar{G}(x ; \xi)]]^{d}-a(w+1)[-\ln [\bar{G}(x ; \xi)]]^{b}\right\} .
\end{aligned}
$$

Using the exponential expansion form for the following term:

$$
\begin{array}{r}
\exp \left[(w+1)\left\{-c[-\ln [\bar{G}(x ; \xi)]]^{d}-a(w+1)[-\ln [\bar{G}(x ; \xi)]]^{b}\right\}\right] \\
=\sum_{i, j=0}^{\infty} \frac{(-1)^{i}(w+1)^{i} a^{j} c^{i-j}}{i ! j !(i-j) !}[-\ln [\bar{G}(x ; \xi)]]^{i d+j(b-d)}
\end{array}
$$

Substituting in (23)

Therefore,

$$
f_{r: n}(x ; \Phi)=\eta_{r} \frac{g(x ; \xi)}{\bar{G}(x ; \xi)}\left\{c d[-\ln [\bar{G}(x ; \xi)]]^{(i+1) d+j(b-d)-1}+a b[-\ln [\bar{G}(x ; \xi)]]^{i d+j(b-d)+b-1}\right\}
$$

where,

$$
\eta_{r}=\frac{1}{B(r, n-r+1)} \sum_{s=0}^{n-r} \sum_{i, j, w=0}^{\infty} \frac{(-1)^{i+s+w}}{i ! j !(i-j) !}\left(\begin{array}{c}
n-r \\
s
\end{array}\right)\left(\begin{array}{c}
r+s-1 \\
w
\end{array}\right)(w+1)^{i} a^{j} c^{i-j} .
$$

In particular, the pdf of the smallest order statistic $X_{1: n}$ is obtained from (24), by substituting $\mathrm{r}=1$

$$
f_{1: n}(x ; \Phi)=\eta_{1} \frac{g(x ; \xi)}{\bar{G}(x ; \xi)}\left\{c d[-\ln [\bar{G}(x ; \xi)]]^{(i+1) d+j(b-d)-1}+a b[-\ln [\bar{G}(x ; \xi)]]^{i d+j(b-d)+b-1}\right\},
$$

where,

$$
\eta_{1}=n \sum_{s=0}^{n-1} \sum_{i, j, w=0}^{\infty} \frac{(-1)^{i+s+w}}{i ! j !(i-j) !}\left(\begin{array}{c}
n-1 \\
s
\end{array}\right)\left(\begin{array}{c}
s \\
w
\end{array}\right)(w+1)^{i} a^{j} c^{i-j} .
$$

Also, the pdf of the largest order statistic $X_{n: n}$ is obtained from (24), by substituting r=n

$$
f_{n: n}(x ; \Phi)=\eta_{n} \frac{g(x ; \xi)}{\bar{G}(x ; \xi)}\left\{c d[-\ln [\bar{G}(x ; \xi)]]^{(i+1) d+j(b-d)-1}+a b[-\ln [\bar{G}(x ; \xi)]]^{i d+j(b-d)+b-1}\right\},
$$

where,

$$
\eta_{n}=n \sum_{i, j, w=0}^{\infty} \frac{(-1)^{i+s+w}}{i ! j !(i-j) !}\left(\begin{array}{c}
n+s-1 \\
w
\end{array}\right)(w+1)^{i} a^{j} c^{i-j}
$$

\subsection{Incomplete Moments, Mean Deviations and Lorenz and Benferroni Curves}

The r-th incomplete moment, say, $m_{r}^{I}(t)$, of the GAW-G distribution is given by

$$
m_{r}^{I}(t)=\int_{0}^{t} x^{r} f(x, \Phi) d x .
$$


We can write from equation (17),

$$
\begin{aligned}
m_{r}^{I}(t)= & \int_{0}^{t} x^{r}\left[\sum_{i, j=0}^{\infty} k \cdot w_{i j}[G(x, \xi)]^{k-1} g(x, \xi)\right. \\
& \left.+\sum_{i, j, m=0}^{\infty}(k+m+1) \cdot w_{i j m}[G(x, \xi)]^{k+m} g(x, \xi)\right] d x .
\end{aligned}
$$

Example 4.5.1 Consider the GAW-uniform distribution discussed in subsection 3.1.

$$
m_{r}^{I}(t)=\sum_{i, j=0}^{\infty} \frac{k}{r+k} \cdot w_{i j} \frac{t^{r+k}}{\theta^{k}}+\sum_{i, j, m=0}^{\infty} \frac{k+m+1}{r+k+m+1} \cdot w_{i j m} \frac{t^{r+k+m+1}}{\theta^{k+m+1}}
$$

The scatterings present in a population is, to some extent, to be measured by the totality of the deviations from a measure of central tendency like the mean or the median. The mean deviations about the mean $\delta_{1}=E\left(\left|X-\mu_{1}^{\prime}\right|\right)$ and median $\delta_{2}=E(|X-M|)$ of $X$ may be used as measures of spread (or dispersion) in a population besides range and standard deviation. They are given by $\delta_{1}=2 \mu_{1}^{\prime} F\left(\mu_{1}^{\prime}\right)-2 m_{1}^{I}\left(\mu_{1}^{\prime}\right)$ and $\delta_{2}=\mu_{1}^{\prime}-2 m_{1}^{I}(M)$,respectively. Here, $\mu_{1}^{\prime}=E(X)$ is to be obtained from (21) with $r=1, F\left(\mu_{1}^{\prime}\right)$ is to calculated from (5), $m_{1}^{I}\left(\mu_{1}^{\prime}\right)$ is the first incomplete function obtained from (25) with $r=1$ and $M$ is the median of $X$ obtained by solving (20) for $u=0.5$.

The Lorenz and Benferroni curves are defined by $L(p)=m_{1}^{I}\left(x_{p}\right) / \mu_{1}^{\prime}$ and $B(p)=m_{1}^{I}\left(x_{p}\right) /\left(p \mu_{1}^{\prime}\right)$, respectively, where $x_{p}=F^{-1}(p)$ can be computed numerically by (20) with $u=p$. These curves have significant roles in demography, economics, insurance, medicine and reliability. For details in this aspect, the readers are referred to Pundir et al.(2005) and references cited therein.

\subsection{Moments of the Residual Life}

The hazard rate, mean residual life, left truncated mean function are some functions related to the residual lifetime of a unit. These functions uniquely determine the cumulative distribution function, $F(x)$. See, for instance, Gupta(1975) and Zoroa et al.(1990).

Definition 4.6.1 Let $X$, be a random variable denoting the lifetime of a unit is at age $t$. Then $X_{t}=X-t \mid X>t$ denotes the remaining lifetime beyond that age $t$.

The cdf $F(x)$ is uniquely determined by the $\mathrm{r}$-th moment of the residual life of $X$ (for $r=1,2, \ldots$ )[Navarro et al.(1998)], and it is given by

$$
\begin{aligned}
m_{r}(t)=E\left[X_{t}\right] & =\frac{1}{\bar{F}(t)} \int_{t}^{\infty}(x-t)^{r} d F(x) \\
& =\frac{1}{1-F(t)} \int_{t}^{\infty}(x-t)^{r} f(x, \Phi) d x
\end{aligned}
$$

In particular, if $r=1$, then $m_{1}(t)$ represents an interesting function called the mean residual life (MRL) function that indicates the expected life length for a unit which is alive at age $t$. The MRL function has wide spectrum of applications in reliability/survival analysis, social studies, biomedical sciences, economics, population study, insurance industry, maintenance and product quality control and product technology.

Example 4.6.1 Consider again the GAW-uniform distribution discussed in subsection 3.1.

$$
\bar{F}(t)=\exp \left[-c\left\{-\ln \left(1-\frac{t}{\theta}\right)\right\}^{d}-a\left\{-\ln \left(1-\frac{t}{\theta}\right)\right\}^{b}\right]
$$

Using (17), we have

$$
\begin{aligned}
\int_{t}^{\theta}(x-t)^{r} f(x, \Phi) d x= & \sum_{i, j=0}^{\infty} \frac{k}{\theta^{k}} \cdot w_{i j} \sum_{u=0}^{r}\left(\begin{array}{l}
u \\
r
\end{array}\right)(-t)^{r-u} \frac{\theta^{u+k}-t^{u+k}}{u+k} \\
& +\sum_{i, j, m=0}^{\infty} \frac{k+m+1}{\theta^{k+m+1}} \cdot w_{i j m} \sum_{u=0}^{r}\left(\begin{array}{l}
u \\
r
\end{array}\right)(-t)^{r-u} \frac{\theta^{u+k+m+1}-t^{u+k+m+1}}{u+k+m+1}
\end{aligned}
$$


For the MRL function,

$$
\begin{aligned}
\int_{t}^{\theta}(x-t) f(x, \Phi) d x= & \sum_{i, j=0}^{\infty} \frac{k}{\theta^{k}} \cdot w_{i j}\left[\frac{\theta^{k+1}-t^{k+1}}{k+1}-\frac{t\left(\theta^{k}-t^{k}\right)}{k}\right] \\
& +\sum_{i, j, m=0}^{\infty} \frac{k+m+1}{\theta^{k+m+1}} \cdot w_{i j m}\left[\frac{\theta^{k+m+2}-t^{k+m+2}}{k+m+2}-\frac{t\left(\theta^{k+m+1}-t^{k+m+1}\right)}{k+m+1}\right]
\end{aligned}
$$

\subsection{Moments of the Reversed Residual Life}

In some life testing aspects, instead of relating uncertainty to the future, it may relate to the past. When the state of a system is observed only at a preassigned inspection time $t$ and if it is found to be at "down" state, then failure lies on the past i.e. the instant in $(0, t)$ at which it has failed. Therefore, study of a notion that is complementary to the residual life, in the sense that it deals with the past time instead of future seems worthwhile [see Di Crescenzo and Longobardi (2002)].

Definition 4.7.1 Let $X$ be a random variable denoting the lifetime of a unit is down at age $t$. Then $\bar{X}_{t}=t-X \mid X<t$ denotes the idle time or inactivity time or reversed residual life of the unit at age $t$.

In case of forensic science, one may be interested in estimating $\bar{X}_{t}$ to have an idea about the exact time of death of a living creature. In Insurance study, it represents the unpaid period of a policy holder due to death. For details, see Block et al.(1998), Chandra and Roy(2001), Maiti and Nanda(2009), and Nanda et al.(2003). The r-th moment of $\bar{X}_{t}$ (for $r=1,2, \ldots)$ is given by

$$
\begin{aligned}
\bar{m}_{r}(t)=E\left[\bar{X}_{t}\right] & =\frac{1}{F(t)} \int_{0}^{t}(t-x)^{r} d F(x) \\
& =\frac{1}{F(t)} \int_{0}^{t}(t-x)^{r} f(x, \Phi) d x
\end{aligned}
$$

In particular, if $r=1$, then $\bar{m}_{1}(t)$ represents a function called the mean idle time or inactivity time (MIT) or reversed residual life (MRRL) function that indicates the expected inactive life length for a unit which is first observed down at age $t$. The properties of MIT function have been explored by Ahmad et al. (2005) and Kayid and Ahmad (2004).

Example 4.7.1 Consider again the GAW-uniform distribution discussed in subsection 3.1.

$$
F(t)=1-\exp \left[-c\left\{-\ln \left(1-\frac{t}{\theta}\right)\right\}^{d}-a\left\{-\ln \left(1-\frac{t}{\theta}\right)\right\}^{b}\right]
$$

Using (17), we have

$$
\begin{aligned}
\int_{0}^{t}(t-x)^{r} f(x, \Phi) d x= & \sum_{i, j=0}^{\infty} \frac{k t^{r+k}}{\theta^{k}} \cdot w_{i j} \sum_{u=0}^{r}(-1)^{u} \frac{\left(\begin{array}{l}
u \\
r
\end{array}\right)}{u+k} \\
& +\sum_{i, j, m=0}^{\infty} \frac{(k+m+1) t^{r+k+m+1}}{\theta^{k+m+1}} \cdot w_{i j m} \sum_{u=0}^{r}(-1)^{u} \frac{\left(\begin{array}{l}
u \\
r
\end{array}\right)}{u+k+m+1}
\end{aligned}
$$

For the MIT (or MRRL) function,

$$
\begin{aligned}
\int_{0}^{t}(t-x) f(x, \Phi) d x= & \sum_{i, j=0}^{\infty} \frac{t^{k+1}}{(k+1) \theta^{k}} \cdot w_{i j} \\
& +\sum_{i, j, m=0}^{\infty} \frac{t^{k+m+2}}{(k+m+2) \theta^{k+m+1}} \cdot w_{i j m}
\end{aligned}
$$

\section{Estimation of Model Parameters}

In this section, the maximum likelihood estimators of the model parameters $\Phi=(a, b, c, d, \xi)$ of GAW-G family from complete samples are derived. Let $X_{1}, X_{2}, \ldots, X_{n}$ be a simple random sample from GAW-G family with observed values 
$x_{1}, x_{2}, \ldots ., x_{n}$. The log likelihood function of (6) is obtained as follows

$$
\begin{gathered}
\ln L(\Phi)=\sum_{i=1}^{n} \ln \left\{\frac{g\left(x_{i} ; \xi\right)}{\bar{G}\left(x_{i} ; \xi\right)}\left\{c d\left[-\ln \left[\bar{G}\left(x_{i} ; \xi\right)\right]\right]^{d-1}+a b\left[-\ln \left[\bar{G}\left(x_{i} ; \xi\right)\right]\right]^{b-1}\right\}\right\} \\
-c \sum_{i=1}^{n}\left[-\ln \left[\bar{G}\left(x_{i} ; \xi\right)\right]\right]^{d}-a \sum_{i=1}^{n}\left[-\ln \left[\bar{G}\left(x_{i} ; \xi\right)\right]\right]^{b} . \\
\ln L(\Phi)=\sum_{i=1}^{n} \ln \left[\frac{g\left(x_{i} ; \xi\right)}{\bar{G}\left(x_{i} ; \xi\right)}\right]+\sum_{i=1}^{n} \ln \left\{c d\left[-\ln \left[\bar{G}\left(x_{i} ; \xi\right)\right]\right]^{d-1}+a b\left[-\ln \left[\bar{G}\left(x_{i} ; \xi\right)\right]\right]^{b-1}\right\} \\
\quad-c \sum_{i=1}^{n}\left[-\ln \left[\bar{G}\left(x_{i} ; \xi\right)\right]\right]^{d}-a \sum_{i=1}^{n}\left[-\ln \left[\bar{G}\left(x_{i} ; \xi\right)\right]\right]^{b} . \\
\ln L(\Phi)=\sum_{i=1}^{n} \ln g\left(x_{i} ; \xi\right)-\sum_{i=1}^{n} \ln \bar{G}\left(x_{i} ; \xi\right)+\sum_{i=1}^{n} \ln \left\{c d\left[-\ln \left[\bar{G}\left(x_{i} ; \xi\right)\right]\right]^{d-1}+a b\left[-\ln \left[\bar{G}\left(x_{i} ; \xi\right)\right]\right]^{b-1}\right\} \\
-c \sum_{i=1}^{n}\left[-\ln \left[\bar{G}\left(x_{i} ; \xi\right)\right]\right]^{d}-a \sum_{i=1}^{n}\left[-\ln \left[\bar{G}\left(x_{i} ; \xi\right)\right]\right]^{b} .
\end{gathered}
$$

For simplicity, let

$$
Z_{i}=c d\left[-\ln \left[\bar{G}\left(x_{i} ; \xi\right)\right]\right]^{d-1}+a b\left[-\ln \left[\bar{G}\left(x_{i} ; \xi\right)\right]\right]^{b-1},
$$

and $\ln L(\Phi)$ to be 1 , then

$$
l=\sum_{i=1}^{n} \ln g\left(x_{i} ; \xi\right)-\sum_{i=1}^{n} \ln \bar{G}\left(x_{i} ; \xi\right)+\sum_{i=1}^{n} \ln Z_{i}-c \sum_{i=1}^{n}\left[-\ln \left[\bar{G}\left(x_{i} ; \xi\right)\right]\right]^{d}-a \sum_{i=1}^{n}\left[-\ln \left[\bar{G}\left(x_{i} ; \xi\right)\right]\right]^{b} .
$$

Differentiating with respect to each parameter and setting the result equals to zero, the maximum likelihood estimators will be obtained. The partial derivatives of $l$ with respect to each parameter are given by

$$
\begin{gathered}
\frac{\partial l}{\partial a}=\sum_{i=1}^{n} \frac{Z_{i a}^{\prime}}{Z_{i}}-\sum_{i=1}^{n}\left[-\ln \left[\bar{G}\left(x_{i} ; \xi\right)\right]\right]^{b}, \\
\frac{\partial l}{\partial b}=\sum_{i=1}^{n} \frac{Z_{i b}^{\prime}}{Z_{i}}-a \sum_{i=1}^{n}\left[-\ln \left[\bar{G}\left(x_{i} ; \xi\right)\right]\right]^{b} \ln \left[-\ln \left[\bar{G}\left(x_{i} ; \xi\right)\right]\right], \\
\frac{\partial l}{\partial c}=\sum_{i=1}^{n} \frac{Z_{i c}^{\prime}}{Z_{i}}-\sum_{i=1}^{n}\left[-\ln \left[\bar{G}\left(x_{i} ; \xi\right)\right]\right]^{d}, \\
\frac{\partial l}{\partial d}=\sum_{i=1}^{n} \frac{Z_{i d}^{\prime}}{Z_{i}}-c \sum_{i=1}^{n}\left[-\ln \left[\bar{G}\left(x_{i} ; \xi\right)\right]\right]^{d} \ln \left[-\ln \left[\bar{G}\left(x_{i} ; \xi\right)\right]\right], \\
\frac{\partial l}{\partial \xi}=\sum_{i=1}^{n} \frac{g^{\prime}\left(x_{i} ; \xi\right)}{g\left(x_{i} ; \xi\right)}+\sum_{i=1}^{n} \frac{g\left(x_{i} ; \xi\right)}{\bar{G}\left(x_{i} ; \xi\right)}\left[1-c d\left[-\ln \left[\bar{G}\left(x_{i} ; \xi\right)\right]\right]^{d-1}-a b\left[-\ln \left[\bar{G}\left(x_{i} ; \xi\right)\right]\right]^{b-1}\right]+\sum_{i=1}^{n} \frac{Z_{i \xi}^{\prime}}{Z_{i}}
\end{gathered}
$$

where

$$
Z_{i a}^{\prime}=\frac{\partial Z_{i}}{\partial a}=b\left[-\ln \left[\bar{G}\left(x_{i} ; \xi\right)\right]\right]^{b-1},
$$




$$
\begin{gathered}
Z_{i b}^{\prime}=\frac{\partial Z_{i}}{\partial b}=a g\left(x_{i} ; \xi\right)\left[1+b \ln \left[G\left(x_{i} ; \xi\right)\right]-b \ln \left[\bar{G}\left(x_{i} ; \xi\right)\right]\right] \frac{\left[G\left(x_{i} ; \xi\right)\right]^{b-1}}{\left[\bar{G}\left(x_{i} ; \xi\right)\right]^{b+1}} \\
Z_{i c}^{\prime}=\frac{\partial Z_{i}}{\partial c}=\operatorname{dg}\left(x_{i} ; \xi\right) \frac{\left[G\left(x_{i} ; \xi\right)\right]^{d-1}}{\left[\bar{G}\left(x_{i} ; \xi\right)\right]^{d+1}} \\
Z_{i d}^{\prime}=\frac{\partial Z_{i}}{\partial d}=\operatorname{cg}\left(x_{i} ; \xi\right)\left[1+d \ln \left[G\left(x_{i} ; \xi\right)\right]-d \ln \left[\bar{G}\left(x_{i} ; \xi\right)\right]\right] \frac{\left[G\left(x_{i} ; \xi\right)\right]^{d-1}}{\left[\bar{G}\left(x_{i} ; \xi\right)\right]^{d+1}}
\end{gathered}
$$

and

$$
Z_{i \xi}^{\prime}=\frac{\partial Z_{i}}{\partial \xi}=\left[c d \frac{\left[G\left(x_{i} ; \xi\right)\right]^{d-1}}{\left[\bar{G}\left(x_{i} ; \xi\right)\right]^{d+1}}+a b \frac{\left[G\left(x_{i} ; \xi\right)\right]^{b-1}}{\left[\bar{G}\left(x_{i} ; \xi\right)\right]^{b+1}}\right]\left[g^{\prime}\left(x_{i} ; \xi\right)+g\left(x_{i} ; \xi\right)\left(\ln \left[G\left(x_{i} ; \xi\right)\right]-\ln \left[\bar{G}\left(x_{i} ; \xi\right)\right]\right)\right]
$$

The maximum likelihood estimates (MLEs) of the model parametersare determined by solving the non-linear equations $\frac{\partial l}{\partial a}=0, \frac{\partial l}{\partial b}=0, \frac{\partial l}{\partial c}=0, \frac{\partial l}{\partial d}=0, \frac{\partial l}{\partial \xi}=0$. These equations cannot be solved analytically but some software's can be used to solve them numerically.

\section{Simulation Study}

In this section, we have conducted simulation study for above mentioned four Generalized Additive Weibull model. We have generated samples of sizes $n=20,40,100$ from each model and parameters have been estimated by the maximum likelihood method. 1000 such repetitions are made to calculate the bias and mean square error (mse) of these estimates using the formula for estimates of any parameter $\eta$ by $\operatorname{Bias}_{\eta}(\hat{\eta})=\frac{1}{1000} \sum_{i=1}^{1000}(\hat{\eta}-\eta)$ and $\operatorname{MSE}_{\eta}(\hat{\eta})=\frac{1}{1000} \sum_{i=1}^{1000}(\hat{\eta}-\eta)^{2}$, respectively. All the computations are made using R-Software.

From the Tables $1-4$, it is observed that

\begin{tabular}{|c|c|c|c|c|c|c|c|c|c|c|}
\hline \multirow[b]{2}{*}{$\mathrm{n}$} & \multicolumn{2}{|c|}{$\theta=0.1$} & \multicolumn{2}{|c|}{$a=1$} & \multicolumn{2}{|c|}{$\mathrm{b}=1$} & \multicolumn{2}{|c|}{$\mathrm{c}=1$} & \multicolumn{2}{|c|}{$\mathrm{d}=1$} \\
\hline & Bias & MSE & Bias & MSE & Bias & MSE & Bias & MSE & Bias & MSE \\
\hline 20 & 0.136 & 0.022 & 4.243 & 24.856 & 3.978 & 24.186 & -0.958 & 1.869 & 1.075 & 21.867 \\
\hline 40 & 0.102 & 0.018 & 3.135 & 10.961 & 3.108 & 15.335 & -0.950 & 1.824 & 0.931 & 14.270 \\
\hline 50 & 0.092 & 0.012 & 2.771 & 9.332 & 2.956 & 12.917 & -0.940 & 0.912 & 0.923 & 9.429 \\
\hline 100 & 0.064 & 0.008 & 8.327 & 5.881 & 2.095 & 12.486 & -0.796 & 0.892 & 0.685 & 8.695 \\
\hline \multirow[t]{2}{*}{300} & 0.059 & 0.007 & 1.442 & 2.389 & 2.018 & 11.620 & -0.781 & 0.819 & 0.264 & 7.486 \\
\hline & \multicolumn{2}{|c|}{$\theta=0.05$} & \multicolumn{2}{|c|}{$a=1$} & \multicolumn{2}{|c|}{$\mathrm{b}=1$} & \multicolumn{2}{|c|}{$c=$} & \multicolumn{2}{|c|}{$\mathrm{d}=1$} \\
\hline $\mathrm{n}$ & Bias & MSE & Bias & MSE & Bias & MSE & Bias & MSE & Bias & MSE \\
\hline 20 & 0.054 & 0.004 & 2.876 & 11.713 & 2.562 & 10.283 & -0.936 & 0.962 & 0.017 & 0.434 \\
\hline 40 & 0.036 & 0.002 & 2.791 & 10.961 & 2.320 & 8.320 & -0.910 & 0.951 & 0.578 & 15.442 \\
\hline \multirow[t]{2}{*}{100} & 0.020 & 0.001 & 2.629 & 8.327 & 1.928 & 7.191 & -0.902 & 0.914 & 0.709 & 23.392 \\
\hline & \multicolumn{2}{|c|}{$\theta=0.05$} & \multicolumn{2}{|c|}{$\mathrm{a}=1.1$} & \multicolumn{2}{|c|}{$b=1$} & & \\
\hline $\mathrm{n}$ & Bias & MSE & Bias & MSE & Bias & MSE & Bias & MSE & Bias & MSE \\
\hline 20 & 0.052 & 0.003 & 2.946 & 13.527 & 2.843 & 22.711 & -9.872 & 0.926 & 0.266 & 3.146 \\
\hline 40 & 0.032 & 0.002 & 2.549 & 11.399 & 2.111 & 11.605 & -9.849 & 0.982 & 0.542 & 9.032 \\
\hline \multirow[t]{2}{*}{100} & 0.018 & 0.001 & 2.385 & 9.682 & 2.117 & 9.671 & -8.453 & 0.981 & 0.381 & 4.320 \\
\hline & \multicolumn{2}{|c|}{$\theta=0.05$} & \multicolumn{2}{|c|}{$a=1$} & \multicolumn{2}{|c|}{$\mathrm{b}=1.5$} & \multicolumn{2}{|c|}{$\mathrm{c}=1$} & \multicolumn{2}{|c|}{$\mathrm{d}=1$} \\
\hline $\mathrm{n}$ & Bias & MSE & Bias & MSE & Bias & MSE & Bias & MSE & Bias & MSE \\
\hline 20 & 0.038 & 0.002 & 3.146 & 15.095 & 0.004 & 15.531 & 0.931 & 0.922 & 0.280 & 2.109 \\
\hline 40 & 0.052 & 0.004 & 3.135 & 14.244 & 0.135 & 16.829 & 0.948 & 1.174 & 0.765 & 2.085 \\
\hline \multirow[t]{2}{*}{100} & 0.073 & 0.006 & 3.339 & 13.547 & -0.214 & 16.052 & 0.963 & 1.362 & 0.231 & 2.304 \\
\hline & \multicolumn{2}{|c|}{$\theta=0.05$} & \multicolumn{2}{|c|}{$a=1$} & \multicolumn{2}{|c|}{$\mathrm{b}=1$} & \multicolumn{2}{|c|}{$\mathrm{c}=1.5$} & \multicolumn{2}{|c|}{$d=1$} \\
\hline $\mathrm{n}$ & Bias & MSE & Bias & MSE & Bias & MSE & Bias & MSE & Bias & MSE \\
\hline 20 & 0.007 & 0.000 & 3.035 & 11.234 & 0.620 & 11.174 & 1.349 & 1.801 & 0.881 & 24.482 \\
\hline 40 & 0.019 & 0.001 & 2.961 & 11.418 & 0.473 & 9.994 & 1.065 & 1.958 & 0.978 & 21.743 \\
\hline 100 & 0.036 & 0.002 & 2.632 & 11.410 & 4.200 & 12.960 & 1.824 & 1.085 & 0.319 & 15.109 \\
\hline
\end{tabular}

1. As sample size $n$ increases, bias decreases. That shows accuracy of the MLE of the parameters,

2. As sample size $n$ increases, MSE decreases. That shows consistency (or preciseness) of the MLE of the parameters.

Table 1. Bias and Mean Square Error (MSE) of the MLE of parameters of GAW-uniform distribution 
Table 2. Bias and Mean Square Error (MSE) of the MLE of parameters of GAW-Gumbel distribution

\begin{tabular}{|c|c|c|c|c|c|c|c|c|c|c|c|c|}
\hline \multirow[b]{2}{*}{$\mathrm{n}$} & \multicolumn{2}{|c|}{$\lambda=0.05$} & \multicolumn{2}{|c|}{$v=0.1$} & \multicolumn{2}{|c|}{$a=1$} & \multicolumn{2}{|c|}{$\mathrm{b}=1$} & \multicolumn{2}{|c|}{$\mathrm{c}=1$} & \multicolumn{2}{|c|}{$\mathrm{d}=1$} \\
\hline & Bias & MSE & Bias & MSE & Bias & MSE & Bias & MSE & Bias & MSE & Bias & MSE \\
\hline 20 & -0.212 & 0.384 & 0.110 & 2.262 & -2.292 & 4.927 & -0.860 & 2.412 & -0.898 & 6.360 & -0.959 & 1.347 \\
\hline 40 & -0.040 & 0.002 & -0.100 & 0.010 & -0.727 & 0.638 & -0.859 & 0.740 & -0.830 & 0.807 & -0.959 & 0.921 \\
\hline 50 & -0.040 & 0.002 & -0.100 & 0.010 & -0.726 & 0.528 & -0.858 & 0.738 & -0.160 & 0.806 & 0.543 & 0.919 \\
\hline 100 & -0.035 & 0.001 & -0.100 & 0.010 & -0.799 & 0.527 & -0.447 & 0.735 & 0.132 & 0.688 & 0.234 & 0.830 \\
\hline \multirow[t]{2}{*}{300} & 0.000 & 0.000 & 0.017 & 0.009 & -0.103 & 0.152 & 0.009 & 0.024 & -0.007 & 0.199 & -0.108 & 0.418 \\
\hline & \multicolumn{2}{|c|}{$\lambda=0.1$} & \multicolumn{2}{|c|}{$v=0.05$} & \multicolumn{2}{|c|}{$a=1$} & \multicolumn{2}{|c|}{$\mathrm{b}=1$} & \multicolumn{2}{|c|}{$\mathrm{c}=1$} & \multicolumn{2}{|c|}{$\mathrm{d}=1$} \\
\hline $\mathrm{n}$ & Bias & MSE & Bias & MSE & Bias & MSE & Bias & MSE & Bias & MSE & Bias & MSE \\
\hline 20 & -0.090 & 0.568 & 0.737 & 2.171 & -2.344 & 13.201 & 2.237 & 4.096 & 3.966 & 12.080 & 6.049 & 15.599 \\
\hline 40 & -0.087 & 0.008 & -0.050 & 0.003 & -0.727 & 0.529 & -0.860 & 0.740 & -0.898 & 0.807 & -0.959 & 0.920 \\
\hline 50 & -0.027 & 0.008 & -0.050 & 0.003 & -0.727 & 0.528 & -0.860 & 0.740 & -0.898 & 0.807 & -0.958 & 0.917 \\
\hline 100 & -0.024 & 0.008 & -0.050 & 0.003 & -0.727 & 0.528 & -0.860 & 0.740 & -0.897 & 0.805 & -0.958 & 0.919 \\
\hline \multirow[t]{2}{*}{300} & -0.017 & 0.008 & -0.050 & 0.003 & -0.727 & 0.528 & -0.859 & 0.738 & -0.896 & 0.804 & -0.957 & 0.916 \\
\hline & \multicolumn{2}{|c|}{$\lambda=0.1$} & \multicolumn{2}{|c|}{$v=0.1$} & \multicolumn{2}{|c|}{$a=1$} & & & & & & \\
\hline $\mathrm{n}$ & Bias & MSE & Bias & MSE & Bias & MSE & Bias & MSE & Bias & MSE & Bias & MSE \\
\hline 20 & -0.050 & 0.003 & -0.100 & 0.010 & -0.840 & 0.707 & -0.877 & 0.770 & -0.943 & 0.889 & -0.889 & 0.791 \\
\hline 40 & -0.050 & 0.003 & -0.100 & 0.010 & -0.840 & 0.707 & -0.876 & 0.768 & -0.942 & 0.888 & -0.888 & 0.790 \\
\hline 50 & -0.050 & 0.003 & -0.100 & 0.010 & -0.832 & 0.692 & -0.810 & 0.656 & -0.941 & 0.886 & -0.849 & 0.720 \\
\hline 100 & -0.050 & 0.003 & -0.100 & 0.010 & -0.831 & 0.691 & -0.810 & 0.655 & -0.878 & 0.771 & -0.848 & 0.720 \\
\hline 300 & -0.050 & 0.003 & -0.100 & 0.010 & -0.831 & 0.691 & -0.809 & 0.655 & -0.876 & 0.768 & -0.848 & 0.719 \\
\hline & & & & & & & & & & & & \\
\hline $\mathrm{n}$ & Bias & MSE & Bias & MSE & Bias & MSE & Bias & MSE & Bias & MSE & Bias & MSE \\
\hline 20 & -0.300 & 0.520 & 0.360 & 0.929 & 1.633 & 10.774 & 2.589 & 18.431 & -2.434 & 17.200 & -0.849 & 9.438 \\
\hline 40 & -0.237 & 0.344 & 0.103 & 0.635 & -1.150 & 7.310 & -0.810 & 15.960 & -1.570 & 15.695 & -0.849 & 4.890 \\
\hline 50 & -0.050 & 0.003 & -0.100 & 0.010 & -0.932 & 0.868 & -0.810 & 0.656 & -0.942 & 0.887 & -0.848 & 0.720 \\
\hline 100 & -0.050 & 0.003 & -0.100 & 0.010 & -0.932 & 0.868 & -0.810 & 0.6 & -0.942 & 0.887 & 0.650 & 0.720 \\
\hline 300 & -0.050 & 0.003 & -0.100 & 0.010 & -0.931 & 0.868 & 0.201 & 0.655 & -0.942 & 0.887 & -0.148 & 0.720 \\
\hline & & & & & & & & & & & & \\
\hline $\mathrm{n}$ & Bias & MSE & Bias & MSE & Bias & MSE & Bias & MSE & Bias & MSE & Bias & MSE \\
\hline 20 & -0.224 & 0.393 & -0.100 & 0.451 & -0.831 & 17.367 & -1.310 & 1.995 & -1.771 & 9.325 & -0.849 & 14.455 \\
\hline 40 & -0.050 & 0.003 & -0.100 & 0.010 & -0.831 & 0.691 & -1.310 & 1.715 & -0.942 & 0.888 & -0.848 & 0.720 \\
\hline 50 & -0.050 & 0.003 & -0.100 & 0.010 & -0.831 & 0.691 & -1.310 & 1.715 & -0.942 & 0.888 & -0.848 & 0.720 \\
\hline 100 & -0.050 & 0.003 & -0.100 & 0.010 & -0.446 & 0.691 & -1.310 & 0.715 & -0.942 & 0.888 & -0.088 & 0.720 \\
\hline 300 & -0.050 & 0.003 & 0.093 & 0.010 & -0.097 & 0.691 & 0.046 & 1.715 & -0.942 & 0.887 & -0.048 & 0.720 \\
\hline & & & & & & & & & & & & \\
\hline $\mathrm{n}$ & Bias & MSE & Bias & MSE & Bias & MSE & Bias & MSE & Bias & MSE & Bias & MSE \\
\hline 20 & -0.274 & 0.298 & 0.257 & 16.507 & 4.805 & 25.439 & 2.739 & 2.420 & -1.443 & 10.866 & -0.849 & 8.988 \\
\hline 40 & -0.050 & 0.003 & -0.100 & 0.010 & -0.832 & 0.692 & -0.810 & 0.656 & -1.443 & 2.082 & -0.849 & 0.720 \\
\hline 50 & -0.050 & 0.003 & -0.100 & 0.010 & -0.832 & 0.691 & -0.810 & 0.656 & -1.441 & 2.081 & -0.848 & 0.720 \\
\hline 100 & -0.050 & 0.003 & -0.100 & 0.010 & -0.831 & 0.691 & -0.809 & 0.655 & -1.358 & 2.079 & -0.849 & 0.719 \\
\hline 300 & -0.050 & 0.003 & -0.100 & 0.010 & -0.831 & 0.690 & -0.809 & 0.655 & -0.442 & 2.078 & 0.083 & 0.719 \\
\hline & & & & & & & & & & & & \\
\hline $\mathrm{n}$ & Bias & MSE & Bias & MSE & Bias & MSE & Bias & MSE & Bias & MSE & Bias & MSE \\
\hline 20 & -0.105 & 0.432 & 3.232 & 12.985 & -1.492 & 5.589 & 5.770 & 24.086 & 2.816 & 7.658 & -1.390 & 2.509 \\
\hline 40 & -0.050 & 0.003 & -0.100 & 0.010 & -0.841 & 0.708 & -0.879 & 0.773 & -0.942 & 0.888 & -1.386 & 1.938 \\
\hline 50 & -0.050 & 0.003 & -0.100 & 0.010 & -0.840 & 0.706 & -0.877 & 0.769 & -0.880 & 0.774 & 1.287 & 1.932 \\
\hline 100 & -0.050 & 0.003 & -0.100 & 0.010 & -0.838 & 0.703 & -0.875 & 0.767 & -0.879 & 0.772 & 1.012 & 1.923 \\
\hline 300 & -0.050 & 0.003 & -0.100 & 0.010 & -0.831 & 0.691 & -0.810 & 0.655 & -0.877 & 0.770 & -0.483 & 1.818 \\
\hline
\end{tabular}


Table 3. Bias and Mean Square Error (MSE) of the MLE of parameters of GAW log-logistic distribution

\begin{tabular}{|c|c|c|c|c|c|c|c|c|c|c|c|c|}
\hline \multirow[b]{2}{*}{$\mathrm{n}$} & \multicolumn{2}{|c|}{$\lambda=0.05$} & \multicolumn{2}{|c|}{$\alpha=0.1$} & \multicolumn{2}{|c|}{$a=1$} & \multicolumn{2}{|c|}{$\mathrm{b}=1$} & \multicolumn{2}{|c|}{$\mathrm{c}=1$} & \multicolumn{2}{|c|}{$\mathrm{d}=1$} \\
\hline & Bias & MSE & Bias & MSE & Bias & MSE & Bias & MSE & Bias & MSE & Bias & MSE \\
\hline 20 & 2.022 & 5.476 & 0.127 & 0.036 & 0.313 & 0.428 & -0.447 & 0.577 & -1.897 & 3.617 & -1.999 & 4.023 \\
\hline 40 & 2.010 & 5.434 & 0.127 & 0.036 & 0.313 & 0.428 & -0.447 & 0.577 & -1.897 & 3.617 & -1.999 & 4.022 \\
\hline 50 & 1.994 & 5.310 & 0.124 & 0.036 & 0.304 & 0.416 & -0.416 & 0.554 & -1.896 & 3.615 & -1.990 & 3.989 \\
\hline 100 & 1.942 & 5.110 & 0.124 & 0.036 & 0.303 & 0.410 & -0.415 & 0.534 & -1.891 & 3.597 & -1.989 & 3.984 \\
\hline \multirow[t]{2}{*}{300} & 1.896 & 4.873 & 0.121 & 0.035 & 0.295 & 0.392 & -0.379 & 0.498 & -1.889 & 3.588 & -1.909 & 3.981 \\
\hline & \multicolumn{2}{|c|}{$\lambda=0.1$} & \multicolumn{2}{|c|}{$\alpha=0.05$} & \multicolumn{2}{|c|}{$a=1$} & \multicolumn{2}{|c|}{$\mathrm{b}=1$} & \multicolumn{2}{|c|}{$\mathrm{c}=1$} & \multicolumn{2}{|c|}{$\mathrm{d}=1$} \\
\hline $\mathrm{n}$ & Bias & MSE & Bias & MSE & Bias & MSE & Bias & MSE & Bias & MSE & Bias & MSE \\
\hline 20 & 1.892 & 4.922 & 0.188 & 0.055 & 0.348 & 0.442 & -0.409 & 0.533 & -1.900 & 3.626 & -2.007 & 4.054 \\
\hline 40 & 1.888 & 4.875 & 0.186 & 0.055 & 0.342 & 0.426 & -0.398 & 0.523 & -1.899 & 3.624 & -2.004 & 4.042 \\
\hline 50 & 1.864 & 4.769 & 0.181 & 0.053 & 0.341 & 0.421 & -0.396 & 0.512 & -1.896 & 3.612 & -2.003 & 4.040 \\
\hline 100 & 1.860 & 4.763 & 0.181 & 0.053 & 0.337 & 0.418 & -0.385 & 0.507 & -1.890 & 3.591 & -2.001 & 4.029 \\
\hline \multirow[t]{2}{*}{300} & 1.851 & 4.671 & 0.176 & 0.051 & 0.336 & 0.396 & -0.361 & 0.490 & -1.888 & 3.585 & -1.999 & 4.021 \\
\hline & \multicolumn{2}{|c|}{$\lambda=0.1$} & \multicolumn{2}{|c|}{$\alpha=0.1$} & \multicolumn{2}{|c|}{$a=1$} & & & & & & \\
\hline $\mathrm{n}$ & Bias & MSE & Bias & MSE & Bias & MSE & Bias & MSE & Bias & MSE & Bias & MSE \\
\hline 20 & 1.926 & 5.081 & 0.138 & 0.039 & 0.370 & 0.464 & -0.432 & 0.556 & -1.893 & 3.604 & -2.010 & 4.063 \\
\hline 40 & 1.876 & 4.839 & 0.131 & 0.037 & 0.360 & 0.437 & -0.402 & 0.521 & -1.892 & 3.599 & -2.009 & 4.037 \\
\hline 50 & 1.842 & 4.663 & 0.124 & 0.037 & 0.350 & 0.434 & -0.376 & 0.495 & -1.890 & 3.592 & -2.008 & 4.026 \\
\hline 100 & 1.820 & 4.597 & 0.118 & 0.035 & 0.343 & 0.428 & -0.372 & 0.491 & -1.884 & 3.570 & -1.994 & 4.004 \\
\hline 300 & 1.815 & 4.558 & 0.116 & 0.033 & 0.328 & 0.421 & -0.352 & 0.487 & -1.878 & 3.550 & -1.992 & 3.993 \\
\hline 100 & 1.820 & 4.597 & 0.131 & 0.037 & 0.343 & 0.421 & -0.352 & 0.491 & -1.884 & 3.570 & -2.008 & 4.026 \\
\hline & & & & & & & & & & & & \\
\hline $\mathrm{n}$ & Bias & MSE & Bias & MSE & Bias & MSE & Bias & MSE & Bias & MSE & Bias & MSE \\
\hline 20 & 1.892 & 4.905 & 0.137 & 0.039 & 0.264 & 0.374 & -0.406 & 0.529 & -1.896 & 3.613 & -2.008 & 4.059 \\
\hline 40 & 1.889 & 4.890 & 0.135 & 0.038 & 0.249 & 0.370 & -0.403 & 0.527 & -1.895 & 3.610 & -2.008 & 4.056 \\
\hline 50 & 1.861 & 4.746 & 0.133 & 0.038 & 0.240 & 0.369 & -0.383 & 0.504 & -1.895 & 3.610 & -2.002 & 4.035 \\
\hline 100 & 1.841 & 4.672 & 0.132 & 0.038 & 0.240 & 0.367 & -0.381 & 0.499 & -1.893 & 3.603 & -2.001 & 4.030 \\
\hline 300 & 1.834 & 4.639 & 0.120 & 0.035 & 0.195 & 0.359 & -0.377 & 0.493 & -1.892 & 3.598 & -1.987 & 3.974 \\
\hline & & & & & & & & & & & & \\
\hline $\mathrm{n}$ & Bias & MSE & Bias & MSE & Bias & MSE & Bias & MSE & Bias & MSE & Bias & MSE \\
\hline 20 & 1.976 & 5.310 & 0.137 & 0.038 & 0.352 & 0.430 & -0.957 & 1.295 & -1.899 & 3.625 & -2.006 & 4.051 \\
\hline 40 & 1.941 & 5.127 & 0.133 & 0.038 & 0.329 & 0.428 & -0.927 & 1.235 & -1.897 & 3.617 & -2.001 & 4.030 \\
\hline 50 & 1.907 & 4.966 & 0.129 & 0.037 & 0.314 & 0.427 & -0.909 & 1.195 & -1.892 & 3.597 & -1.996 & 4.012 \\
\hline 100 & 1.862 & 4.761 & 0.119 & 0.035 & 0.306 & 0.417 & -0.887 & 1.145 & -1.890 & 3.592 & -1.987 & 3.977 \\
\hline 300 & 1.802 & 4.498 & 0.117 & 0.034 & 0.302 & 0.413 & -0.865 & 1.090 & -1.889 & 3.587 & -1.985 & 3.968 \\
\hline & & & & & & & & & & & & \\
\hline $\mathrm{n}$ & Bias & MSE & Bias & MSE & Bias & MSE & Bias & MSE & Bias & MSE & Bias & MSE \\
\hline 20 & 1.938 & 5.129 & 0.135 & 0.038 & 0.346 & 0.432 & -0.411 & 0.544 & -2.397 & 5.763 & -2.003 & 4.037 \\
\hline 40 & 1.927 & 5.039 & 0.132 & 0.037 & 0.345 & 0.428 & -0.410 & 0.535 & -2.395 & 5.755 & -2.002 & 4.035 \\
\hline 50 & 1.882 & 4.864 & 0.132 & 0.037 & 0.336 & 0.427 & -0.403 & 0.524 & -2.394 & 5.750 & -2.000 & 4.028 \\
\hline 100 & 1.876 & 4.837 & 0.131 & 0.037 & 0.321 & 0.426 & -0.401 & 0.521 & -2.394 & 5.748 & -2.000 & 4.027 \\
\hline 300 & 1.875 & 4.822 & 0.125 & 0.036 & 0.314 & 0.400 & -0.394 & 0.517 & -2.392 & 5.743 & -1.994 & 4.001 \\
\hline & & & & & & & & & & & & \\
\hline $\mathrm{n}$ & Bias & MSE & Bias & MSE & Bias & MSE & Bias & MSE & Bias & MSE & Bias & MSE \\
\hline 20 & 1.858 & 4.756 & 0.139 & 0.039 & 0.384 & 0.445 & -0.392 & 0.509 & -1.903 & 3.638 & -2.514 & 6.343 \\
\hline 40 & 1.819 & 4.569 & 0.139 & 0.039 & 0.374 & 0.439 & -0.368 & 0.482 & -1.894 & 3.605 & -2.512 & 6.335 \\
\hline 50 & 1.814 & 4.506 & 0.135 & 0.038 & 0.363 & 0.439 & -0.367 & 0.475 & -1.891 & 3.597 & -2.512 & 6.333 \\
\hline 100 & 1.801 & 4.493 & 0.133 & 0.038 & 0.358 & 0.410 & -0.361 & 0.472 & -1.891 & 3.597 & -2.505 & 6.301 \\
\hline 300 & 1.798 & 4.490 & 0.128 & 0.037 & 0.295 & 0.402 & -0.347 & 0.469 & -1.886 & 3.579 & -2.492 & 6.236 \\
\hline
\end{tabular}


Table 4. Bias and Mean Square Error (MSE) of the MLE of parameters of GAW-Burr XII distribution

\begin{tabular}{|c|c|c|c|c|c|c|c|c|c|c|c|c|}
\hline \multirow[b]{2}{*}{$\mathrm{n}$} & \multicolumn{2}{|c|}{$\alpha=0.05$} & \multicolumn{2}{|c|}{$\theta=0.1$} & \multicolumn{2}{|c|}{$a=1$} & \multicolumn{2}{|c|}{$b=1$} & \multicolumn{2}{|c|}{$\mathrm{c}=1$} & \multicolumn{2}{|c|}{$\mathrm{d}=1$} \\
\hline & Bias & MSE & Bias & MSE & Bias & MSE & Bias & MSE & Bias & MSE & Bias & MSE \\
\hline 20 & 2.163 & 5.467 & 0.808 & 0.795 & -1.786 & 3.343 & -1.782 & 3.294 & 0.088 & 0.141 & -0.949 & 0.926 \\
\hline 40 & 127 & 5.250 & .801 & 0.786 & -1.763 & 3.258 & -1.771 & 3.273 & 0.076 & 0.140 & 0.946 & 0.920 \\
\hline 50 & 032 & 4.836 & .789 & 0.758 & -1.758 & 3.242 & -1.761 & 3.237 & 0.071 & 0.139 & -0.946 & 0.918 \\
\hline 100 & 987 & 4.382 & .783 & 0.728 & -1.572 & 3.157 & -1.728 & 3.201 & 0.068 & 0.108 & -0.912 & 0.917 \\
\hline \multirow[t]{2}{*}{300} & 020 & 2.624 & 0.428 & 0.689 & -1.287 & 2.451 & -1.048 & 2.941 & 0.021 & 0.089 & -0.821 & 0.894 \\
\hline & \multicolumn{2}{|c|}{$\alpha=0.1$} & \multicolumn{2}{|c|}{$\theta=0.05$} & \multicolumn{2}{|c|}{$a=1$} & \multicolumn{2}{|c|}{$\mathrm{b}=1$} & \multicolumn{2}{|c|}{$c=1$} & \multicolumn{2}{|c|}{$\mathrm{d}=1$} \\
\hline $\mathrm{n}$ & Bias & MSE & Bias & MSE & Bias & MSE & Bias & MSE & Bias & SE & Bias & MSE \\
\hline 20 & 2.085 & 5.104 & 0.872 & 0.893 & -1.726 & 3.397 & -1.789 & 3.301 & 0.085 & 154 & 0.953 & 0.929 \\
\hline 40 & 965 & 4.992 & 0.861 & 0.884 & -1.814 & 3. & -1.785 & 3.2 & 0.085 & 8 & -0.950 & 0.923 \\
\hline 50 & 63 & 4.980 & 0.847 & 0.862 & -1.798 & 3.2 & -1.779 & 3.2 & 0.072 & 8 & 948 & 0.919 \\
\hline 100 & 962 & 4.213 & 0.729 & 0.814 & -1.240 & 3.0 & -1.621 & 2.870 & 0.067 & 3 & .911 & 0.824 \\
\hline \multirow[t]{2}{*}{300} & 1.254 & 3.785 & 0.639 & 0.725 & -1.768 & 2.089 & -1.029 & 1.962 & 0.006 & 0.002 & -0.716 & 0.124 \\
\hline & \multicolumn{2}{|c|}{$\alpha=0.1$} & \multicolumn{2}{|c|}{$\theta=0.1$} & \multicolumn{2}{|c|}{$a=1$} & & & & & & \\
\hline $\mathrm{n}$ & Bias & MSE & Bias & MSE & Bias & MSE & Bias & MSE & Bias & MSE & Bias & MSE \\
\hline 20 & 14 & 5.248 & 0.824 & 0.828 & -1.817 & 3.459 & -1.801 & 3.339 & 0.109 & 7 & 960 & 0.943 \\
\hline 40 & 037 & 4.891 & 0.822 & 0.819 & -1.807 & 3.416 & -1.774 & 3.252 & 0.100 & 0.145 & -0.957 & 0.942 \\
\hline 50 & 033 & 4.860 & 0.792 & 0.780 & -1.775 & 3.326 & -1.761 & 3.208 & 0.095 & 0.144 & -0.939 & 0.937 \\
\hline 100 & 924 & 3.421 & 0.761 & 0.764 & -1.722 & 2.982 & -1.624 & 3.024 & 0.090 & 0.143 & -0.921 & 0.903 \\
\hline 300 & 1.263 & 3.105 & 0.611 & 0.578 & -1.423 & 1.826 & -1.072 & 2.879 & 0.077 & 0.085 & -0.815 & 0.819 \\
\hline & & & & & & & & & & & & \\
\hline $\mathrm{n}$ & Bias & MSE & Bias & MSE & Bias & MSE & Bias & MSE & ias & MSE & Bias & MSE \\
\hline 20 & 2.121 & 5.259 & 0.799 & 0.774 & -1.866 & 3.642 & -1.773 & 3.240 & 0.078 & 45 & 950 & 922 \\
\hline 40 & 05 & 5. & 0. & & & & & & & & & 920 \\
\hline 50 & 87 & 5.0 & 0.790 & 0. & 1 & & 54 & & 59 & & & 0.906 \\
\hline 100 & 25 & 4.5 & 0.780 & 0.7 & 25 & & 82 & & 0.065 & & 10 & 0.895 \\
\hline 300 & 1.529 & 3.562 & 0.681 & 0.613 & -1.802 & 3.025 & -1.047 & 2.227 & 0.024 & 0.096 & .842 & 0.682 \\
\hline & & & & & & & & & & & & \\
\hline $\mathrm{n}$ & Bias & MSE & Bias & MSE & Bias & MSE & Bias & MSE & Bias & MSE & Bias & MSE \\
\hline 20 & 106 & 5.197 & 0.815 & 0.802 & 1.685 & 3.3 & -2.281 & 5.3 & 0.096 & 0 & 7 & 0.918 \\
\hline 40 & 779 & 5.077 & 0.798 & 0.801 & -1.811 & 3.297 & -2.272 & 5.263 & 0.095 & 0.1 & -0.947 & 0.917 \\
\hline 50 & 19 & 4.962 & 0.785 & 0.779 & -1.058 & 3.25 & -2.266 & 5.2 & 0.083 & 0.1 & -0.945 & 0.915 \\
\hline 100 & 1.994 & 4.8 & 0.725 & 0.772 & -1.772 & 2.9 & -1.998 & 4.9 & 0.077 & 0. & -0.935 & 0.914 \\
\hline 300 & 1.657 & 3.485 & 0.631 & 0.724 & -1.760 & 2.008 & -1.492 & 3.06 & 0.076 & 0.139 & -0.821 & 0.854 \\
\hline & & & & & & & & & & & & \\
\hline $\mathrm{n}$ & Bias & MSE & Bias & MSE & Bias & MSE & Bias & MSE & Bias & MSE & Bias & MSE \\
\hline 20 & 102 & 5.185 & 0.824 & 0.828 & 808 & & -1.804 & 33 & 0.409 & 4 & 962 & 0.945 \\
\hline 40 & 2.025 & 4.804 & 0.820 & 0.817 & -1.789 & & 04 & 8 & -0.402 & & & 0.936 \\
\hline 50 & 2.021 & 4.7 & 0.815 & 0.811 & -1.789 & & -1.778 & & -0.393 & & & 0.932 \\
\hline 100 & 1.929 & & 0.792 & & & & & & -0.390 & & 12 & 0.908 \\
\hline 300 & 1.283 & 2.956 & 0.562 & 0.496 & -1.186 & 2.028 & -1.447 & 2.973 & -0.354 & 0.211 & -0.812 & 0.870 \\
\hline & & & & & & & & & & & & \\
\hline $\mathrm{n}$ & Bias & MSE & Bias & MSE & Bias & MSE & Bias & MS & Bias & MSE & Bias & MSE \\
\hline 20 & 109 & 5.223 & 0.812 & 0.810 & -1.779 & & -1.779 & & 0.093 & & -1.451 & 2.127 \\
\hline 40 & 00 & 5.1 & & & -1.773 & 3.3 & -1.777 & 3.2 & 0.073 & 0. & -1.450 & 2.122 \\
\hline 50 & 061 & 4.9 & 0.800 & 0.775 & -1.758 & & -1.756 & 3.1 & 0.065 & 0.139 & -1.444 & 2.106 \\
\hline 100 & 87 & 4.8 & 0.794 & 0.769 & -1.689 & 3.1 & -1.682 & 2.9 & 0.051 & 0.138 & -1.378 & 2.078 \\
\hline 300 & 1.028 & 3.049 & 0.789 & 0.724 & -1.284 & 2.459 & -1.235 & 2.186 & 0.018 & 0.028 & -1.178 & 1.982 \\
\hline
\end{tabular}

\section{Application of GAW-G Family to Real Data}

In this section, we fit the GAW-Uniform model to a real data set obtained from from Andrews and Herzberg (1985) and the original source is Barlow et al. (1984) and have been shown in Table 5. Histogram shows that the data set is positively skewed. Al-Aqtash et al.(2014) fitted this data to the Gumbel-Weibull Distribution. We have fitted this data set with the GAW-Uniform distribution. The estimated values of the parameters were $\hat{\theta}=7.890, \hat{a}=3.389, \hat{b}=0.767, \hat{c}=3.769$, and $\hat{d}=1.361, \log$-likelihood $=-94.249$ and AIC $=198.498$. Histogram and fitted GAW-Uniform curve to data have been shown in Figure 2. 
Table 5. Kevlar 49/epoxy strands failure times data (pressure at 90 percentage)

\begin{tabular}{lllllllllllllllllll}
\hline 0.01 & 0.01 & 0.02 & 0.02 & 0.02 & 0.03 & 0.03 & 0.04 & 0.05 & 0.06 & 0.07 & 0.07 & 0.08 & 0.09 & 0.09 & 0.10 & 0.10 & 0.11 & 0.11 \\
0.12 & 0.13 & 0.18 & 0.19 & 0.20 & 0.23 & 0.24 & 0.24 & 0.29 & 0.34 & 0.35 & 0.36 & 0.38 & 0.40 & 0.42 & 0.43 & 0.52 & 0.54 & 0.56 \\
0.60 & 0.60 & 0.63 & 0.65 & 0.67 & 0.68 & 0.72 & 0.72 & 0.72 & 0.73 & 0.79 & 0.79 & 0.80 & 0.80 & 0.83 & 0.85 & 0.90 & 0.92 & 0.95 \\
0.99 & 1.00 & 1.01 & 1.02 & 1.03 & 1.05 & 1.10 & 1.10 & 1.11 & 1.15 & 1.18 & 1.20 & 1.29 & 1.31 & 1.33 & 1.34 & 1.40 & 1.43 & 1.45 \\
1.50 & 1.51 & 1.52 & 1.53 & 1.54 & 1.54 & 1.55 & 1.58 & 1.60 & 1.63 & 1.64 & 1.80 & 1.80 & 1.81 & 2.02 & 2.05 & 2.14 & 2.17 & 2.33 \\
3.03 & 3.03 & 3.34 & 4.20 & 4.69 & 7.89 & & & & & & & & & & & & & \\
\hline
\end{tabular}

Table 6. Summarized results of fitting different distributions for Kevlar 49/epoxy strands failure times data

\begin{tabular}{lccc}
\hline Distribution & Estimate of the parameter & Log-likelihood & AIC \\
\hline Exponential & $\hat{\lambda}=0.976$ & -103.479 & 208.958 \\
Gumbel-Weibull & $\hat{\beta}=1.806, \hat{\sigma}=3.271, \hat{\lambda}=0.207, \hat{a}=0.920$ & -100.23 & 208.500 \\
GAW-Uniform & $\hat{\theta}=7.890, \hat{a}=3.389, \hat{b}=0.767, \hat{c}=3.769, \hat{d}=1.361$ & -94.249 & 198.498 \\
\hline
\end{tabular}
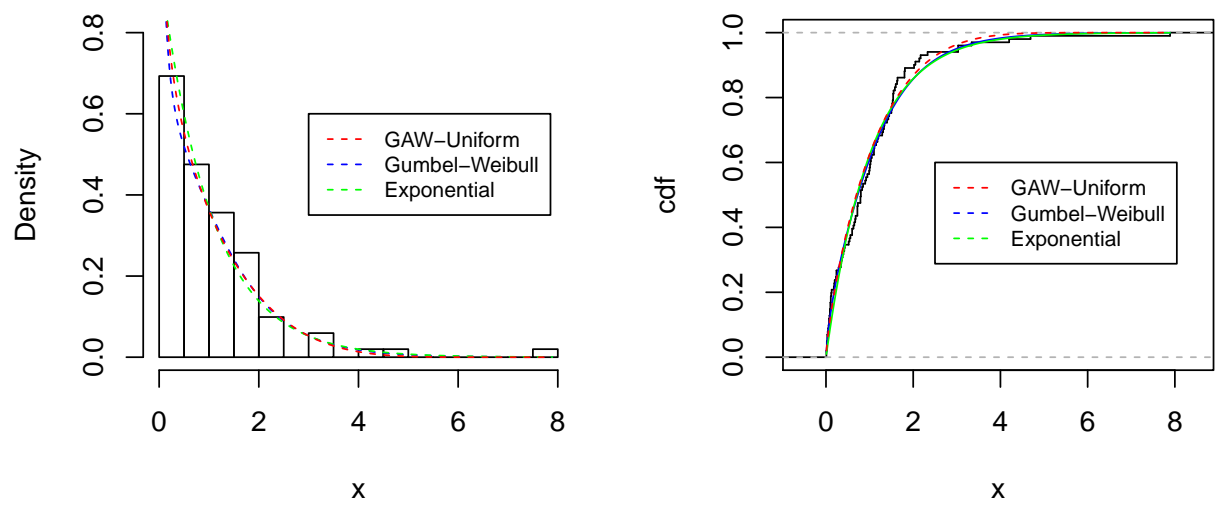

Figure 2. Plots of the Histogram, estimated pdf and cdf for failure times data (pressure at 90 percentage)

\section{Concluding Remarks}

We have introduced and studied a new generalized family of distributions, called the generalized additive Weibull-G (GAW-G) distribution. The GAW- G family generalizes the Weibull-G family [see, Cordeiro et al.(2015)] and includes several new distributions. Properties of the GAW-G family include: an expansion for the density function and expressions for the quantile function, moment generating function, ordinary moments, incomplete moments, mean deviations, Lorenz and Benferroni curves, reliability properties including mean residual life and mean inactivity time, and order statistics. Four new distributions, namely, GAW-Uniform, GAW-Gumble, GAW-Log logistic and GAW-Burr XII are defined and discussed in some details. The maximum likelihood method is employed to estimate the model parameters. Simulation study has been conducted to study the accuracy and consistency of the MLE of the parameters. A real data set is used to demonstrate the flexibility of distribution belonging to the introduced family. These special models give better fits than other models. We hope the findings of the paper will be quite useful for the practitioners in various fields of probability, statistics and applied sciences.

\section{Acknowledgements}

The authors would like to thank the anonymous referees and editor for their valuable comments and suggestions that helped the manuscript to improve a lot.

\section{References}

Ahmad, I., Kayid, M., \& Pellerey, F. (2005). Further results involving the MIT order and the IMIT class. Probability in the Engineering and Informational Science, 19, 377-395. https://doi.org/10.1017/S0269964805050229

Al-Aqtash, R., Lee, C., \& Famoye, F. (2014). Gumbel-Weibull distribution: Properties and applications. Journal of Modern Applied Statistical Methods, 13(2), 201-225. 
Alexander, C., Cordeiro, G. M., Ortega, E. M. M., \& Sarabia, J. M. (2012). Generalized beta-generated distributions. Computational Statistics and Data Analysis, 56, 1880-1897. https://doi.org/10.1016/j.csda.2011.11.015

Alzaatreh, A., Lee, C., \& Famoye, F. (2013). A new method for generating families of continuous distributions. Metron, 71, 63-79. https://doi:10.1007/s40300-013-0007-y

Andrews, D. F., \& Herzberg, A. M. (1985). Prognostic variables for survival in a randomized comparison of treatments for prostatic cancer. Data, Springer New York, 261-274.

Barlow, D. H., Cohen, A. S., ..., \& Di Nardo, P. A. (1984). Panic and generalized anxiety disorders: Nature and treatment. Behavior Therapy, 15(5), 431-449. https://doi.org/10.1016/S0005-7894(84)80048-9

Bennett, S. (1983). Log-logistic regression models for survival data. Journal of the Royal Statistical Society, Series C (Applied Statistics), 32(2), 165-171. https://doi: 10.2307/2347295

Block, H., Savits, T., \& Singh, H. (1998). The reversed hazard rate function. Probability in the Engineering and Informational Sciences, 12, 69C90. https://doi.org/10.1017/S0269964800005064

Bourguignon, M., Silva, R. B., \& Cordeiro, G. M. (2014). The Weibull-G family of probability distributions. Journal of Data Science, 12, 53-68.

Burr, I. W. (1942): Cumulative frequency functions, The Annals of Mathematical Statistics, 13(2), 215-232. https:// doi:10.1214/aoms/1177731607

Chandra, N. K., \& Roy, D. (2001). Some results on reversed hazard rate. Probability in the Engineering and Informational Sciences, 15, 95C102. https://doi.org/10.1017/S0269964801151077

Cordeiro, G. M., \& de Castro, M. (2011). A new family of generalized distributions. Journal of Statistical Computation and Simulation, 81(7), 883-898. http://dx.doi.org/10.1080/00949650903530745

Cordeiro, G. M., Ortega, M. M. E., \& Ramires, T. G. (2015). A new generalized Weibull family of distributions: mathematical properties and applications. Journal of Statistical Distributions and Applications, 2(13), 25. https://doi:10.1186/s40488015-0036-6

Cordeiro, G. M., Alizadeh, M., \& Marinho, P. R. D. (2016). The type I half-logistic family of distributions. Journal of Statistical Computation and Simulation, 86(4),707-728. http://dx.doi.org/10.1080/00949655.2015.1031233

Di Crescenzo, A., \& Longobardi, M. (2002). Entropy-based measure of uncertainty in past lifetime distribution. Journal of Applied Probability, 39, 434C 440. https://doi.org/10.1017/S002190020002266X

Eugene, N., Lee, C., \& Famoye, F. (2002). Beta-normal distribution and its applications. Communications in StatisticsTheory and Methods, 31(4), 497-512. http://dx.doi.org/10.1081/STA-120003130

Flajolet, P., \& Odlyzko, A. (1990). Singularity analysis of generating functions. SIAM Journal on discrete mathematics, 3(2), 216-240. http://dx.doi.org/10.1137/0403019

Gupta, R. C. (1975). On characterization of distributions by conditional expectations. Communications in Statistics: Simulation and Computation, 4(1), 99-103. http://dx.doi.org/10.1080/03610927508827230

Hassan, A. S., \& Hemeda, S. E. (2016). A New Family of Additive Weibull-Generated Distributions. International Journal of Mathematics and its Applications, 4(2), 151-164.

Kayid, M., \& Ahmad, I. (2004). On the mean inactivity time ordering with reliability applications. Probability in the Engineering and Informational Science, 18, 395-409. https://doi.org/10.1017/S0269964804183071

Lemonte, A. J., Cordeiro, G. M., \& Ortega, M. M. E. (2104). On the additive Weibull distribution. Communications in Statistics-Theory and Methods, 43(10-12), 2066-2080. http://dx.doi.org/10.1080/03610926.2013.766343

Maiti, S. S., \& Nanda, A. K. (2009). A loglikelihood-based shape measure of past lifetime distribution. Calcutta Statistical Association Bulletin, 61, 303-320. http://journals.sagepub.com/doi/10.1177/0008068320090116

Nanda, A. K., Singh, H., Misra, N., \& Paul, P. (2003). Reliability properties of reversed residual lifetime. Communications in Statistics: Theory and Methods, 32(10), 2031C2042. http://dx.doi.org/10.1081/STA-120023264

Navarro, J., Franco, M., \& Ruiz, J. M. (1998). Characterization through moments of the residual life and conditional spacing. Sankhya, 60, Series A, 36-48. http://www.jstor.org/stable/25051181

Pundir, S., Arora, S., \& Jain, K. (2005). Bonferroni curve and the related statistical inference. Statistics and Probability Letters, 75, 140-150. https://doi.org/10.1016/j.spl.2005.05.024 
Ward, M. (1934). The representation of Stirling's numbers and Stirling's polynomials as sums of factorials. American Journal of Mathematics, 56(1/4), 87-95. http://www.jstor.org/stable/2370916

Zografos, K., \& Balakrishnan, N. (2009). On families of beta- and generalized gamma-generated distributions and associated inference. Statistical Methodology, 6, 344-362. https://doi.org/10.1016/j.stamet.2008.12.003

Zoroa, P., Ruiz, J. M., \& Marin, J. (1990). A characterization based on conditional expectations. Communications in Statistics: Theory and Methods, 19, 3127-3135. http://dx.doi.org/10.1080/03610929008830368

\section{Copyrights}

Copyright for this article is retained by the author(s), with first publication rights granted to the journal.

This is an open-access article distributed under the terms and conditions of the Creative Commons Attribution license (http://creativecommons.org/licenses/by/4.0/). 